\title{
Bifundamental Fuzzy 2-Sphere and Fuzzy Killing Spinors ${ }^{\star}$
}

\author{
Horatiu NASTASE ${ }^{\dagger}$ and Constantinos PAPAGEORGAKIS $\ddagger$ \\ † Instituto de Física Teórica, UNESP-Universidade Estadual Paulista, \\ R. Dr. Bento T. Ferraz 271, Bl. II, Sao Paulo 01140-070, SP, Brazil \\ E-mail: nastase@ift.unesp.br \\ $\ddagger$ Department of Mathematics, King's College London, \\ The Strand, London WC2R 2LS, UK \\ E-mail: costis.papageorgakis@kcl.ac.uk
}

Received March 26, 2010, in final form July 09, 2010; Published online July 20, 2010 doi:10.3842/SIGMA.2010.058

\begin{abstract}
We review our construction of a bifundamental version of the fuzzy 2-sphere and its relation to fuzzy Killing spinors, first obtained in the context of the ABJM membrane model. This is shown to be completely equivalent to the usual (adjoint) fuzzy sphere. We discuss the mathematical details of the bifundamental fuzzy sphere and its field theory expansion in a model-independent way. We also examine how this new formulation affects the twisting of the fields, when comparing the field theory on the fuzzy sphere background with the compactification of the 'deconstructed' (higher dimensional) field theory.
\end{abstract}

Key words: noncommutative geometry; fuzzy sphere; field theory

2010 Mathematics Subject Classification: 81T75; 81T30

\section{Introduction and Motivation}

Noncommutative geometry is a tool that finds numerous applications in the description of a wide range of physical systems. A celebrated example appearing in String Theory is in terms of the polarisation phenomenon discovered by Myers, in which $N \mathrm{D} p$-branes in the presence of transverse Ramond-Ramond flux distribute themselves onto the surface of a higher-dimensional sphere [1]. The physics of the simplest case are captured by a $\mathrm{U}(N)$ theory, with the solution involving fuzzy 2 -spheres $[2,3,4]$. These are related to families of Hermitian matrices obeying the $\mathrm{SU}(2)$ algebra

$$
\left[X^{i}, X^{j}\right]=2 i \epsilon^{i j k} X^{k}
$$

The $X^{i}$ enter the physics as ground state solutions to the equations of motion via (1.1). Then their commutator action on the space of all $N \times N$ matrices organises the matrices into representations of $\mathrm{SU}(2) \simeq \mathrm{SO}(3)$. An important aspect of the geometry of the fuzzy 2-sphere involves the construction of fuzzy (matrix) spherical harmonics in $\mathrm{SU}(2)$ representations, which approach the space of all classical $S^{2}$ spherical harmonics in the limit of large matrices [3]. This construction of fuzzy spherical harmonics allows the analysis of fluctuations in a non-Abelian theory of $\mathrm{D} p$-branes to be expressed at large $N$ in terms of an Abelian higher dimensional theory. This describes a $\mathrm{D}(p+2)$ brane wrapping the sphere, with $N$ units of worldvolume magnetic flux. At finite $N$ the higher dimensional theory becomes a noncommutative $\mathrm{U}(1)$ with a UV cutoff $[5,6,7,8]$.

\footnotetext{
*This paper is a contribution to the Special Issue "Noncommutative Spaces and Fields". The full collection is available at http://www.emis.de/journals/SIGMA/noncommutative.html
} 
In this article we review a novel realisation of the fuzzy 2-sphere involving bifundamental matrices. The objects that crucially enter the construction are discrete versions of Killing spinors on the sphere $[9,10]^{1}$. The motivation is similar to the above and comes from the study of the model recently discovered by Aharony, Bergman, Jafferis and Maldacena (ABJM) describing the dynamics of multiple parallel M2-branes on a $\mathbb{Z}_{k}$ M-theory orbifold [11], which followed the initial investigations of Bagger-Lambert and Gustavsson (BLG) [12, 13, 14, 15].

The ABJM theory is an $\mathcal{N}=6$ superconformal Chern-Simons-matter theory with $\mathrm{SO}(6)$ R-symmetry and gauge group $\mathrm{U}(N) \times \mathrm{U}(\bar{N})$. The two Chern-Simons (CS) terms have equal but opposite levels $(k,-k)$ and the matter fields transform in the bifundamental representation. One can use the inverse CS level $1 / k$ as a coupling constant to perform perturbative calculations. At $k=1$ the theory is strongly coupled and describes membranes in flat space. For $k=1,2$ the supersymmetry and R-symmetry are nonperturbatively enhanced to $\mathcal{N}=8$ and $\mathrm{SO}(8)$ respectively $[11,16,17]$. It is then possible to use this action to investigate aspects of the $\mathrm{AdS}_{4} / \mathrm{CFT}_{3}$ duality, with the role of the 't Hooft coupling played by $\lambda=\frac{N}{k}$. The action of the $\mathbb{Z}_{k}$ orbifold on the $\mathbb{C}^{4}$ space transverse to the M2's is such that taking $k \rightarrow \infty$ corresponds to shrinking the radius of the M-theory circle and entering a IIA string theory regime.

Of particular interest are the ground-state solutions of the maximally supersymmetric massive deformation of ABJM found by Gomis, Rodríguez-Gómez, Van Raamsdonk and Verlinde $\left(\right.$ GRVV) $[18]^{2}$. The theory still has a $\mathrm{U}(N) \times \mathrm{U}(\bar{N})$ gauge group and $\mathcal{N}=6$ supersymmetry but conformal invariance is lost and the R-symmetry is broken down to $\mathrm{SU}(2) \times \mathrm{SU}(2) \times \mathrm{U}(1)$. Its vacua are expected to describe a configuration of M2-branes blowing up into spherical M5branes in the presence of transverse flux through a generalisation of the Myers effect. At $k=1$ these solutions should have a dual description in terms of the $\frac{1}{2}$-BPS M-theory geometries with flux found in [20, 21].

Interestingly, the matrix part of the above ground-state equation is given by the following simple relation, which we will refer to as the GRVV algebra ${ }^{3}$ :

$$
G^{\alpha}=G^{\alpha} G_{\beta}^{\dagger} G^{\beta}-G^{\beta} G_{\beta}^{\dagger} G^{\alpha},
$$

where $G^{\alpha}$ are $N \times \bar{N}$ and $G_{\alpha}^{\dagger}$ are $\bar{N} \times N$ matrices respectively. Given that the Myers effect for the M2-M5 system should employ a 3-dimensional surface, one might initially expect this to represent the defining relation for a fuzzy 3 -sphere. Moreover, the explicit irreducible solutions of (1.2) satisfy $G^{\alpha} G_{\alpha}^{\dagger}=1$, which seems to suggest the desired fuzzy 3 -sphere structure.

However, we will see that the requisite $\mathrm{SO}(4)$ R-symmetry, that would be needed for the existing fuzzy $S^{3}$ construction of Guralnik and Ramgoolam (GR) [24, 25, 26], is absent in this case. As was also shown in [9], the GR fuzzy $S^{3}$ construction implies the following algebra

$$
\begin{aligned}
& \epsilon^{m n p q} X_{n}^{+} X_{p}^{-} X_{q}^{+}=2\left(\frac{(r+1)(r+3)+1}{r+2}\right) X_{m}^{+}, \\
& \epsilon^{m n p q} X_{n}^{-} X_{p}^{+} X_{q}^{-}=2\left(\frac{(r+1)(r+3)+1}{r+2}\right) X_{m}^{-},
\end{aligned}
$$

which must be supplemented with the sphere condition

$$
X_{m} X_{m}=X_{m}^{+} X_{m}^{-}+X_{m}^{-} X_{m}^{+}=\frac{(r+1)(r+3)}{2} \equiv N
$$

and the constraints

$$
X_{m}^{+} X_{n}^{+}=X_{m}^{-} X_{n}^{-}=0 .
$$

\footnotetext{
${ }^{1}$ The work in [9] was carried out in collaboration with S. Ramgoolam.

${ }^{2}$ The mass-deformed theory was also presented in [19].

${ }^{3}$ The same defining matrix equation appears while looking for BPS funnel solutions in the undeformed ABJM theory and first appeared as such in [22]. Its relation to the M2-M5 system was also investigated in [23].
} 
Here $r$ defines a representation of $\mathrm{SO}(4) \simeq \mathrm{SU}(2) \times \mathrm{SU}(2)$ by $\mathcal{R}_{r}^{+}$and $\mathcal{R}_{r}^{-}$, with labels $\left(\frac{r+1}{2}, \frac{r-1}{2}\right)$ and $\left(\frac{r-1}{2}, \frac{r+1}{2}\right)$ respectively for the two groups, and the $X_{m}^{ \pm}$are constructed from gamma matrices. Even though the algebra (1.3) looks similar to the GRVV algebra (1.2), they coincide only in the 'fuzziest' case with $r=1$, i.e. the BLG $\mathcal{A}_{4}$-algebra, which in the Van Raamsdonk $\mathrm{SU}(2) \times \mathrm{SU}(2)$ reformulation [27] is

$$
R^{2} X^{m}=-i k \epsilon^{m n p q} X^{n} X^{\dagger p} X^{q} .
$$

This fact suggests that equation (1.2) does not describe a fuzzy $S^{3}$. Furthermore, the perturbative calculations that lead to the above equation are valid at large $k$, where the ABJM theory is describing IIA String Theory instead of M-theory and as a result a D2-D4 bound state in some nontrivial background.

In the following, we will review how solutions to equation (1.2) actually correspond to a fuzzy 2 -sphere, albeit in a realisation involving bifundamental instead of the usual adjoint matrices, by constructing the full spectrum of spherical harmonics. This is equivalent to the usual construction in terms of the $\mathrm{SU}(2)$ algebra (1.1). In fact there is a one-to-one correspondence between the representations of the $\mathrm{SU}(2)$ algebra $X_{i}$ and the representations in terms of bifundamental matrices. We will also show how the matrices $G^{\alpha}$, which are solutions of the GRVV algebra up to gauge transformations, correspond to fuzzy Killing spinors on the sphere, recovering the usual Killing spinors in the large $N$ limit.

The purpose of this article is to present the mathematical aspects of the above construction in a completely model-independent way and highlight some of its features simply starting from (1.2). The reader who is interested in the full background and calculations in the context of the ABJM model is referred to [9, 10], where an analysis of small fluctuations around the ground-states at large $N, k$ showed that they can be organised in terms of a $\mathrm{U}(1)$ theory on $\mathbb{R}^{2,1} \times S^{2}$, consistent with an interpretation as a D4-brane in Type IIA. The full 3 -sphere expected from M-theory then appeared as the large $N, k=1$ limit of a fuzzy Hopf fibration, $S^{1} / \mathbb{Z}_{k} \hookrightarrow S_{F}^{3} / \mathbb{Z}_{k} \stackrel{\pi}{\rightarrow} S_{F}^{2}$, in which the M-theory circle $S^{1} / \mathbb{Z}_{k}$ is fibred over the noncommutative sphere base, $S_{F}^{2}$.

We also discuss how this bifundamental formulation affects the twisting of the fields when 'deconstructing' a higher dimensional field theory. This is achieved by studying the field theory around a fuzzy sphere background, where the twisting is necessary in order to preserve supersymmetry. Even though the twisting is usually described in the context of compactifying the higher dimensional 'deconstructed' theory, we show how this naturally arises from the bifundamental fuzzy sphere field theory point of view.

The rest of this paper is organised as follows. In Section 2 we give the harmonic decomposition of the GRVV matrices and relate them to the fuzzy supersphere. In Section 3 we present a oneto-one map between the adjoint and bifundamental fuzzy sphere constructions, while in Section 4 we establish that connection in terms of the fuzzy Hopf fibration and define the fuzzy version of Killing spinors on $S^{2}$. We then discuss the resulting 'deconstruction' of higher dimensional field theories on the 2-sphere, specifically the issue of twisting of the fields in order to preserve supersymmetry. In Section 5 we review the process and discuss the differences between the adjoint and bifundamental cases, while in Section 6 we briefly discuss a particular application by summarising the results of $[9,10]$. We conclude with some closing remarks in Section 7 .

\section{Constructing the fluctuation expansion}

Notation. In this section, we will denote by $k, l, m, n$ the matrix indices/indices of states in a vector space, while keeping $i, j=1, \ldots, 3$ as vector indices on the fuzzy $S^{2}$. We will also use $j$ for the $\mathrm{SU}(2)$ spin and $Y_{l m}$ for $S^{2}$ spherical harmonics, following the standard notation. The distinction should be clear by the context. 


\subsection{Ground-state matrices and symmetries}

We begin by writing the ground-state solutions to (1.2), found in [18] and given by

$$
\begin{aligned}
& \left(G^{1}\right)_{m, n}=\sqrt{m-1} \delta_{m, n} \\
& \left(G^{2}\right)_{m, n}=\sqrt{(N-m)} \delta_{m+1, n} \\
& \left(G_{1}^{\dagger}\right)_{m, n}=\sqrt{m-1} \delta_{m, n} \\
& \left(G_{2}^{\dagger}\right)_{m, n}=\sqrt{(N-n)} \delta_{n+1, m}
\end{aligned}
$$

Using the decomposition of the above complex into real coordinates

$$
G^{1}=X^{1}+i X^{2}, \quad G^{2}=X^{3}+i X^{4},
$$

one easily sees that these satisfy

$$
\sum_{p=1}^{4} X_{p} X^{p} \equiv G^{\alpha} G_{\alpha}^{\dagger}=N-1,
$$

which at first glance would seem to indicate a fuzzy $S^{3}$ structure. However, note that in the above $G^{1}=G_{1}^{\dagger}$ for the ground-state solution. With the help of (2.2) this results in $X_{2}=0$, which is instead indicative of a fuzzy $S^{2}$.

As usual in the case of fuzzy sphere constructions, the matrices $G^{\alpha}$ will be used to construct both the symmetry operators (as bilinears in $G, G^{\dagger}$, also acting on $G^{\alpha}$ themselves) as well as fuzzy coordinates, used to expand in terms of spherical harmonics on the fuzzy sphere.

\subsection{1 $G G^{\dagger}$ relations}

As a first step towards uncovering the $S^{2}$ structure we calculate the $G G^{\dagger}$ bilinears

$$
\begin{aligned}
\left(G^{1} G_{1}^{\dagger}\right)_{m, n} & =(m-1) \delta_{m n}, \\
\left(G^{2} G_{2}^{\dagger}\right)_{m n} & =(N-m) \delta_{m n}, \\
\left(G^{1} G_{2}^{\dagger}\right)_{m n} & =\sqrt{(m-1)(N-m+1)} \delta_{m, n+1}, \\
\left(G^{2} G_{1}^{\dagger}\right)_{m n} & =\sqrt{(N-m) m} \delta_{m+1, n}, \\
\left(G^{\alpha} G_{\alpha}^{\dagger}\right)_{m n} & =(N-1) \delta_{m n} .
\end{aligned}
$$

Defining $J_{\beta}^{\alpha}=G^{\alpha} G_{\beta}^{\dagger}$ we get the following commutation relation

$$
\left[J_{\beta}^{\alpha}, J_{\nu}^{\mu}\right]=\delta_{\beta}^{\mu} J_{\nu}^{\alpha}-\delta_{\nu}^{\alpha} J_{\beta}^{\mu} \text {. }
$$

These are commutation relations of the generators of $\mathrm{U}(2)$. Then the $J_{i}=\left(\tilde{\sigma}_{i}\right)_{\beta}^{\alpha} J_{\alpha}^{\beta}$ are the generators of $\mathrm{SU}(2)$ that result in the usual formulation of the fuzzy ${ }^{4} S^{2}$, in terms of the algebra

$$
\left[J_{i}, J_{j}\right]=2 i \epsilon_{i j k} J_{k} .
$$

The trace $J \equiv J_{\alpha}^{\alpha}=N-1$ is a trivial $\mathrm{U}(1) \simeq \mathrm{U}(2) / \mathrm{SU}(2)$ generator, commuting with everything else.

${ }^{4}$ Note that more correctly, we should have written $J^{\alpha}{ }_{\beta}=G^{\alpha} G_{\beta}^{\dagger}$ and

$$
J_{i}=\left(\tilde{\sigma}_{i}\right)^{\alpha}{ }_{\beta} J_{\alpha}^{\beta}=\left(\sigma_{i}\right)_{\beta}{ }^{\alpha} J^{\beta}{ }_{\alpha},
$$

but in the following we will stick to the notation $J_{\beta}^{\alpha}$. The kind of matrix multiplication that one has will be made clear from the context. 


\subsection{2 $G^{\dagger} G$ relations}

Next, we calculate the $G^{\dagger} G$ combinations

$$
\begin{aligned}
\left(G_{1}^{\dagger} G^{1}\right)_{m n} & =(m-1) \delta_{m n}, \\
\left(G_{2}^{\dagger} G^{2}\right)_{m n} & =(N-m+1) \delta_{m n}-N \delta_{m 1} \delta_{n 1}, \\
\left(G_{1}^{\dagger} G^{2}\right)_{m n} & =\sqrt{(m-1)(N-m)} \delta_{m+1, n}, \\
\left(G_{2}^{\dagger} G^{1}\right)_{m n} & =\sqrt{(m-2)(N-m+1)} \delta_{m, n+1}, \\
\left(G_{\alpha}^{\dagger} G^{\alpha}\right)_{m n} & =N \delta_{m n}-N \delta_{m 1} \delta_{n 1}
\end{aligned}
$$

and define $\bar{J}_{\alpha}^{\beta}=G_{\alpha}^{\dagger} G^{\beta}$. The commutation relations for the above then form another copy of $\mathrm{U}(2)$

$$
\left[\bar{J}_{\beta}^{\alpha}, \bar{J}_{\nu}^{\mu}\right]=\delta_{\beta}^{\mu} \bar{J}_{\nu}^{\alpha}-\delta_{\nu}^{\alpha} \bar{J}_{\beta}^{\mu}
$$

and similarly, $\bar{J}_{i}=\left(\tilde{\sigma}_{i}\right)_{\beta}^{\alpha} \bar{J}_{\alpha}^{\beta}$ once again satisfy the usual SU(2) algebra ${ }^{5}$, for another fuzzy $S^{2}$

$$
\left[\bar{J}_{i}, \bar{J}_{j}\right]=2 i \epsilon_{i j k} \bar{J}_{k} .
$$

The trace

$$
(\bar{J})_{m n}=\left(\bar{J}_{\alpha}^{\alpha}\right)_{m n}=N \delta_{m n}-N \delta_{m 1} \delta_{n 1},
$$

which is a $\mathrm{U}(1) \simeq \mathrm{U}(2) / \mathrm{SU}(2)$ generator, commutes with the $\mathrm{SU}(2)$ generators $\bar{J}_{i}$, though as a matrix does not commute with the generators $J_{2}^{1}$ and $J_{1}^{2}$ of the first set of $\mathrm{SU}(2)$ generators.

At this point, it seems that we have two $\mathrm{SU}(2)$ 's, i.e. $\mathrm{SO}(4) \simeq \mathrm{SU}(2) \times \mathrm{SU}(2)$ as expected for a 3-sphere, even though we have not yet shown that these are proper space symmetries: We have only found that the $J, \bar{J}$ satisfy a certain symmetry algebra. In fact, we will next see that these are not independent but rather combine into a single $\mathrm{SU}(2)$.

\subsubsection{Symmetry acting on bifundamental $(N, \bar{N})$ matrices}

All the $(N, \bar{N})$ bifundamental scalar matrices are of the type $G, G G^{\dagger} G, G G^{\dagger} G G^{\dagger} G$, ... The simplest such terms are the $G^{\alpha}$ matrices themselves, the action of the symmetry generators on which we will next investigate.

It is easy to check that the matrices $G^{\alpha}$ satisfy

$$
G^{1} G_{2}^{\dagger} G^{2}-G^{2} G_{2}^{\dagger} G^{1}=G^{1}, \quad G^{2} G_{1}^{\dagger} G^{1}-G^{1} G_{1}^{\dagger} G^{2}=G^{2} .
$$

Using the definitions of $J_{i}$ and $\bar{J}_{i}$, we find

$$
J_{i} G^{\alpha}-G^{\alpha} \bar{J}_{i}=\left(\tilde{\sigma}_{i}\right)_{\beta}^{\alpha} G^{\beta} .
$$

The $G^{1}, G^{2}$ transform like the $(1,0)$ and $(0,1)$ column vectors of the spin- $\frac{1}{2}$ representation with the $J$ 's and $\bar{J}$ 's matrices in the $\mathfrak{u}(N) \times \mathfrak{u}(\bar{N})$ Lie algebra.

\footnotetext{
${ }^{5}$ Again, note that we should have written $\bar{J}_{\alpha}{ }^{\beta}=G_{\alpha}^{\dagger} G^{\beta}$ which emphasises that for $\bar{J}$, the lower index is the first matrix index, and

$$
\bar{J}_{i}=\left(\tilde{\sigma}_{i}\right)^{\alpha}{ }_{\beta} \bar{J}_{\alpha}{ }^{\beta}=\left(\sigma_{i}\right)_{\beta}{ }^{\alpha} \bar{J}_{\alpha}{ }^{\beta},
$$

which emphasises that as matrices, the $\bar{J}_{i}$ are defined with the Pauli matrices, whereas $J_{i}$ was defined with their transpose. However, we will again keep the notation $\bar{J}_{\alpha}^{\beta}$.
} 
By taking Hermitian conjugates in (2.5), we find that the antibifundamental fields, $G_{\alpha}^{\dagger}$, transform as

$$
G_{\alpha}^{\dagger} J_{i}-\bar{J}_{i} G_{\alpha}^{\dagger}=G_{\beta}^{\dagger}\left(\tilde{\sigma}_{i}\right)_{\alpha}^{\beta} .
$$

Therefore the $G^{\alpha}, G_{\alpha}^{\dagger}$ form a representation when acted by both $J_{i}$ and $\bar{J}_{i}$, but neither symmetry by itself gives a representation for $G^{\alpha}, G_{\alpha}^{\dagger}$. This means that the geometry we will be constructing from bifundamental fluctuation modes has a single $\mathrm{SU}(2)$ symmetry, as opposed to two. Equations (2.5) and (2.6) imply relations giving transformations between $J_{i}$ and $\bar{J}_{i}$, thus showing they represent the same symmetry

$$
G_{\gamma}^{\dagger} J_{i} G^{\gamma}=(N+1) \bar{J}_{i}, \quad G^{\gamma} \bar{J}_{i} G_{\gamma}^{\dagger}=(N-2) J_{i} .
$$

Writing the action of the full $\mathrm{SU}(2) \times \mathrm{U}(1)$ on the $G^{\alpha}$, including the $\mathrm{U}(1)$ trace $\bar{J}$, we obtain

$$
J_{\beta}^{\alpha} G^{\gamma}-G^{\gamma} \bar{J}_{\beta}^{\alpha}=\delta_{\beta}^{\gamma} G^{\alpha}-\delta_{\beta}^{\alpha} G^{\gamma},
$$

while taking Hermitian conjugates of (2.7) we obtain the $\mathrm{U}(2)$ transformation of $G_{\alpha}^{\dagger}$,

$$
\bar{J}_{\beta}^{\alpha} G_{\gamma}^{\dagger}-G_{\gamma}^{\dagger} J_{\beta}^{\alpha}=-\delta_{\gamma}^{\alpha} G_{\beta}^{\dagger}+\delta_{\beta}^{\alpha} G_{\gamma}^{\dagger} .
$$

The consequence of the above equations is that $G^{\alpha}$ has charge 1 under the U(1) generator $\bar{J}$. Thus a global U(1) symmetry action on $G^{\alpha}$ does not leave the solution invariant, and we need to combine with the action of $\bar{J}$ from the gauge group to obtain an invariance.

We next turn to the construction of fuzzy spherical harmonics out of $G^{\alpha}$.

\subsection{Fuzzy $S^{2}$ harmonics from $\mathrm{U}(N) \times \mathrm{U}(\bar{N})$ with bifundamentals}

All bifundamental matrices of $\mathrm{U}(N) \times \mathrm{U}(\bar{N})$, are maps between two different vector spaces. On the other hand, products of the bilinears $G G^{\dagger}$ and $G^{\dagger} G$ are adjoint matrices mapping back to the same vector space. Thus, the basis of 'fuzzy spherical harmonics' on our fuzzy sphere will be constructed out of all possible combinations: $\mathrm{U}(N)$ adjoints like $G G^{\dagger}, G G^{\dagger} G G^{\dagger}, \ldots, \mathrm{U}(\bar{N})$ adjoints like $G^{\dagger} G, G^{\dagger} G G^{\dagger} G, \ldots$, and bifundamentals like $G, G G^{\dagger} G, \ldots$ and $G^{\dagger}, G^{\dagger} G G^{\dagger}, \ldots$

\subsubsection{The adjoint of $\mathrm{U}(N)$}

Matrices like $G G^{\dagger}$ act on an $N$ dimensional vector space that we call $\mathbf{V}^{+}$. Thus the space of linear maps from $\mathbf{V}^{+}$back to itself, $\operatorname{End}\left(\mathbf{V}_{+}\right)$, is the adjoint of the $\mathrm{U}(N)$ factor in the $\mathrm{U}(N) \times \mathrm{U}(\bar{N})$ gauge group and $G G^{\dagger}$ are examples of matrices belonging to it. The space $\mathbf{V}^{+}$ forms an irreducible representation of $\mathrm{SU}(2)$ of $\operatorname{spin} j=\frac{N-1}{2}$, denoted by $V_{N}$

$$
\mathbf{V}^{+}=V_{N} .
$$

The set of all operators of the form $G G^{\dagger}, G G^{\dagger} G G^{\dagger}, \ldots$ belong in $\operatorname{End}\left(\mathbf{V}_{+}\right)$and can be expanded in a basis of 'fuzzy spherical harmonics' defined using the $\mathrm{SU}(2)$ structure. Through the $\mathrm{SU}(2)$ generators $J_{i}$ we can form the fuzzy spherical harmonics as

$$
Y^{0}=1, \quad Y_{i}^{1}=J_{i}, \quad Y_{\left(\left(i_{1} i_{2}\right)\right)}^{2}=J_{\left(\left(i_{1}\right.\right.} J_{\left.\left.i_{2}\right)\right)}, \quad Y_{\left(\left(i_{1} \cdots i_{l}\right)\right)}^{l}=J_{\left(\left(i_{1} \cdots J_{\left.\left.i_{l}\right)\right)} .\right.\right.}
$$

In the above, the brackets $\left(\left(i_{1} \cdots i_{l}\right)\right)$ denote traceless symmetrisation. The complete space of $N \times N$ matrices can be expanded in the fuzzy spherical harmonics with $0 \leq l \leq 2 j=N-1$. One indeed checks that

$$
N^{2}=\sum_{l=0}^{2 j}(2 l+1) \text {. }
$$


Then, a general matrix in the adjoint of $\mathrm{U}(N)$ can be expanded as

$$
A=\sum_{l=0}^{N-1} \sum_{m=-l}^{l} a^{l m} Y_{l m}\left(J_{i}\right),
$$

where

$$
Y_{l m}\left(J_{i}\right)=\sum_{i} f_{l m}^{\left(\left(i_{1} \cdots i_{l}\right)\right)} J_{i_{1}} \cdots J_{i_{l}} .
$$

The $Y_{l m}\left(J_{i}\right)$ become the usual spherical harmonics in the 'classical' limit, when $N \rightarrow \infty$ and the cut-off in the angular momentum is removed.

In conclusion, all the matrices of $\mathrm{U}(N)$ can be organised into irreps of $\mathrm{SU}(2)$ constructed out of $J_{i}$, which form the fuzzy spherical harmonics $Y_{l m}\left(J_{i}\right)$.

\subsubsection{The adjoint of $\mathrm{U}(\bar{N})$}

In a fashion similar to the $\mathrm{U}(N)$ case, the matrices $G^{\dagger} G, G^{\dagger} G G^{\dagger} G, \ldots$, are linear endomorphisms of $\mathbf{V}^{-}$. These matrices are in the adjoint of the $\mathrm{U}(\bar{N})$ factor of the $\mathrm{U}(N) \times \mathrm{U}(\bar{N})$ gauge group, and will be organised into irreps of the $\mathrm{SU}(2)$ constructed out of $\bar{J}_{i}$.

However, we now have a new operator: We have already noticed in (2.4) that the U(1) generator $\bar{J}$ is nontrivial. We can express it as

$$
\bar{J}=G_{\alpha}^{\dagger} G^{\alpha}=N-N \bar{E}_{11} .
$$

This means that $\operatorname{End}\left(\mathbf{V}_{-}\right)$contains in addition to the identity matrix, the matrix $\bar{E}_{11}$ which is invariant under $\mathrm{SU}(2)$. If we label the basis states in $\mathbf{V}^{-}$as $\left|e_{k}^{-}\right\rangle$with $k=1, \ldots, N$, then $\bar{E}_{11}=\left|e_{1}^{-}\right\rangle\left\langle e_{1}^{-}\right|$. This in turn means that $\mathbf{V}^{-}$is a reducible representation

$$
\mathbf{V}^{-}=V_{N-1}^{-} \oplus V_{1}^{-} \text {. }
$$

The first direct summand is the irrep of $\mathrm{SU}(2)$ with dimension $N-1$ while the second is a onedimensional irrep. Indeed, one checks that the $\bar{J}_{i}$ 's annihilate the state $\left|e_{1}^{-}\right\rangle$, which is necessary for the identification with the one-dimensional irrep to make sense.

As a result, the space $\operatorname{End}\left(\mathbf{V}^{-}\right)$decomposes as follows

$$
\operatorname{End}\left(\mathbf{V}^{-}\right)=\operatorname{End}\left(V_{N-1}^{-}\right) \oplus \operatorname{End}\left(V_{1}^{-}\right) \oplus \operatorname{Hom}\left(V_{N-1}^{-}, V_{1}^{-}\right) \oplus \operatorname{Hom}\left(V_{1}^{-}, V_{N-1}^{-}\right),
$$

that is, the matrices split as $M_{\mu \nu}=\left(M_{i j}, M_{11}, M_{1 i}, M_{i 1}\right)$. The first summand has a decomposition in terms of another set of fuzzy spherical harmonics

$$
Y_{l m}\left(\bar{J}_{i}\right)=\sum_{i} f_{l m}^{\left(\left(i_{1} \cdots i_{l}\right)\right)} \bar{J}_{i_{1}} \cdots \bar{J}_{i_{l}}
$$

for $l$ going from 0 to $N-2$, since

$$
(N-1)^{2}=\sum_{l=0}^{N-2}(2 l+1) .
$$

This gives only matrices in the $(N-1)$ block, i.e. the $\operatorname{End}\left(V_{N-1}^{-}\right)$. The second summand is just one matrix transforming in the trivial irrep, $\bar{E}_{11}$. The remaining two $N-1$ dimensional spaces of matrices cannot be expressed as products of $\bar{J}_{i}$. They are spanned by

$$
\bar{E}_{1 k}=\left|e_{1}^{-}\right\rangle\left\langle e_{k}^{-}\left|\equiv g_{1 k}^{--}, \quad \bar{E}_{k 1}=\right| e_{k}^{-}\right\rangle\left\langle e_{1}^{-}\right| \equiv g_{k 1}^{--},
$$


which are like spherical harmonics for $\operatorname{Hom}\left(V_{N-1}^{-}, V_{1}^{-}\right) \oplus \operatorname{Hom}\left(V_{1}^{-}, V_{N-1}^{-}\right)$. They transform in the $N-1$ dimensional irrep of SU(2) under the adjoint action of $\bar{J}_{i}$ and are zero mode eigenfunctions of the $\mathrm{U}(1)$ symmetry operator $\bar{J}$.

Therefore, one can expand a general matrix in the adjoint of $\mathrm{U}(\bar{N})$ as

$$
\bar{A}=\bar{a}_{0} \bar{E}_{11}+\sum_{l=0}^{N-2} \sum_{m=-l}^{l} \bar{a}_{l m} Y_{l m}\left(\bar{J}_{i}\right)+\sum_{k=2}^{N} b_{k} g_{1 k}^{--}+\sum_{k=2}^{N} \bar{b}_{k} g_{k 1}^{--}
$$

and note that we could have replaced $\bar{E}_{11}$ with the U(1) generator $\bar{J}$ by redefining $\bar{a}_{0}$ and $\bar{a}_{00}$.

In the large $N$ limit the $Y_{l m}\left(\bar{J}_{i}\right)$ become the ordinary spherical harmonics of $S^{2}$, just like $Y_{l m}\left(J_{i}\right)$. There are order $N^{2}$ of these modes, which is appropriate as the fuzzy $S^{2}$ can roughly be thought of as a 2-dimensional space with each dimension discretised in $N$ units. The mode $\bar{a}_{0}, b_{k}$ and $\bar{b}_{k}$ can be neglected at large $N$, as they have much less than $N^{2}$ degrees of freedom.

\subsubsection{SU(2) harmonic decomposition of bifundamental matrices}

As in the case of the $\mathrm{U}(\bar{N})$ matrices, the bifundamental matrices of the form $G, G G^{\dagger} G, \ldots$ giving physical fluctuating fields, are not enough to completely fill $\operatorname{Hom}\left(\mathbf{V}^{-}, \mathbf{V}^{+}\right)$. Given the decomposition $\mathbf{V}^{-}=V_{N-1}^{-} \oplus V_{1}^{-}$, we decompose $\operatorname{Hom}\left(\mathbf{V}^{-}, \mathbf{V}^{+}\right)$as

$$
\operatorname{Hom}\left(\mathbf{V}^{-}, \mathbf{V}^{+}\right)=\operatorname{Hom}\left(V_{N-1}^{-}, V_{N}^{+}\right) \oplus \operatorname{Hom}\left(V_{1}^{-}, V_{N}^{+}\right)
$$

i.e. the matrices $M_{\mu \nu}$ as $\left(M_{i \nu}, M_{1 \nu}\right)$. The first summand has dimension $N(N-1)$, while the second has dimension $N$ and forms an irreducible representation of $\mathrm{SU}(2)$.

Since the $V_{N-1}^{-}$and $V_{N}^{+}$are irreps of SU(2) we can label the states with the eigenvalue of $\bar{J}_{3}, J_{3}$ respectively. Given our normalisation of the $\mathrm{SU}(2)$ generators in $(2.3)$, the usual spin is $\frac{J_{3}^{\max }}{2}$. The matrices in $\operatorname{Hom}\left(V_{N-1}^{-}, V_{N}^{+}\right)$are of the form $\left|e_{m}^{+}\right\rangle\left\langle e_{n}^{-}\right|$, where $m=\frac{-N+1}{2}, \frac{-N+3}{2}, \ldots, \frac{N^{2}-1}{2}$, $n=\frac{-N+2}{2}, \frac{-N+4}{2}, \ldots, \frac{N-2}{2}$ denote the eigenvalues of $\frac{J_{3}}{2}$. These are spanned by matrices of the form $G\left(\bar{J}_{i_{1}}\right)\left(\bar{J}_{i_{2}}\right) \cdots\left(\bar{J}_{i_{l}}\right)$, i.e. the matrix $G$ times matrices in $\operatorname{End}\left(V_{N-1}^{-}\right)$.

The operators in $\operatorname{Hom}\left(V_{N-1}^{-}, V_{N}^{+}\right)$transform in representations of $\operatorname{spin} l+\frac{1}{2}$ for $l=0, \ldots, N-2$. The dimensions of these representations correctly add up to

$$
\sum_{l=0}^{N-2}(2 l+2)=N(N-1) .
$$

This then gives the $\mathrm{SU}(2)$ decomposition of $\operatorname{Hom}\left(V_{N-1}^{-}, V_{N}^{+}\right)$as

$$
\operatorname{Hom}\left(V_{N-1}^{-}, V_{N}^{+}\right)=\bigoplus_{l=0}^{N-2} V_{l+1 / 2}
$$

On the other hand, matrices $\left|e_{k}^{+}\right\rangle\left\langle e_{1}^{-}\right| \equiv \hat{E}_{k 1} \in \operatorname{Hom}\left(V_{1}^{-}, V_{N}^{+}\right)$cannot be written in terms of the $G^{\prime}$ 's and $G^{\dagger}$ 's alone, because $G^{\alpha}$ acting on $\left|e_{1}^{-}\right\rangle$gives zero. The index $k$ runs over the $N$ states in $\mathbf{V}^{+}$. Here $\hat{E}_{k 1}$ are eigenfunctions of the operator $\bar{E}_{11}$ with unit charge,

$$
\hat{E}_{k 1} \bar{E}_{11}=\hat{E}_{k 1} \text {. }
$$

Combining all of the above, the bifundamental fluctuations $r^{\alpha}$ can be expanded as follows

$$
r^{\alpha}=r_{\beta}^{\alpha} G^{\beta}+\sum_{k=1}^{N} t_{k}^{\alpha} \hat{E}_{k 1}
$$


with

$$
r_{\beta}^{\alpha}=\sum_{l=0}^{N-2} \sum_{m=-l}^{l}\left(r^{l m}\right)_{\beta}^{\alpha} Y_{l m}\left(J_{i}\right) .
$$

We further decompose $r_{\beta}^{\alpha}$ into a trace and a traceless part and define

$$
s_{\beta}^{\alpha}=r_{\beta}^{\alpha}-\frac{1}{2} \delta_{\beta}^{\alpha} r_{\gamma}^{\gamma}, \quad r=r_{\gamma}^{\gamma}, \quad T^{\alpha}=t_{k}^{\alpha} \hat{E}_{k 1} .
$$

Thus the complete expansion of $r^{\alpha}$ is given simply in terms of

$$
r^{\alpha}=r G^{\alpha}+s_{\beta}^{\alpha} G^{\beta}+T^{\alpha} .
$$

We could equivalently have written

$$
r^{\alpha}=\sum_{l=0}^{N-2} \sum_{m=-l}^{l}\left(r^{l m}\right)_{\beta}^{\alpha} G^{\beta} Y_{l m}\left(\bar{J}_{i}\right)+\sum_{k=1}^{N} t_{k}^{\alpha} \hat{E}_{k 1}
$$

using the spherical harmonics in $\bar{J}$ in (2.8). In the following, we will choose, without loss of generality, to work with (2.9).

Until now we have focused on matrices in $\operatorname{Hom}\left(\mathbf{V}^{-}, \mathbf{V}^{+}\right)$but the case of $\operatorname{Hom}\left(\mathbf{V}^{+}, \mathbf{V}^{-}\right)$is similar. The matrices $G^{\dagger}, G^{\dagger} G G^{\dagger}, \ldots$ will also form a representation of $\mathrm{SU}(2)$ given by $\bar{J} \sim G^{\dagger} G$, times a $G^{\dagger}$ matrix. Once again one needs to add an extra $T_{\alpha}^{\dagger}=\left(t_{k}^{\alpha}\right)^{*} \hat{F}_{1 k}$ fluctuation in order to express the matrices $\hat{F}_{1 k} \equiv\left|e_{1}^{-}\right\rangle\left\langle e_{k}^{+}\right| \in \operatorname{Hom}\left(V_{N}^{+}, V_{1}^{-}\right)$. In fact, the result for the complete fluctuating field can be obtained by taking a Hermitian conjugate of (2.9), yielding

$$
r_{\alpha}^{\dagger}=G_{\alpha}^{\dagger} r+G_{\beta}^{\dagger} s_{\alpha}^{\beta}+T_{\alpha}^{\dagger}
$$

\section{$2.3 \quad$ Fuzzy superalgebra}

The matrices $G^{\alpha}$ and $J_{i}$ can be neatly packaged into supermatrices which form a representation of the orthosymplectic Lie superalgebra $\operatorname{OSp}(1 \mid 2)$. The supermatrix is nothing but the embedding of the $N \times \bar{N}$ matrices into $\mathrm{U}(2 N)$. The adjoint fields live in the 'even subspace', while the bifundamentals in the 'odd subspace'. For a generic supermatrix

$$
M=\left(\begin{array}{cc}
A & B \\
C & D
\end{array}\right)
$$

the superadjoint operation is

$$
M^{\ddagger}=\left(\begin{array}{cc}
A^{\dagger} & C^{\dagger} \\
-B^{\dagger} & D^{\dagger}
\end{array}\right) .
$$

For Hermitian supermatrices this is

$$
X=\left(\begin{array}{cc}
A & B \\
-B^{\dagger} & D
\end{array}\right)
$$

with $A=A^{\dagger}$ and $D=D^{\dagger}[28]$. This gives the definition of the supermatrices

$$
\mathbf{J}_{i}=\left(\begin{array}{cc}
J_{i} & 0 \\
0 & \bar{J}_{i}
\end{array}\right) \quad \text { and } \quad \mathbf{J}_{\alpha}=\left(\begin{array}{cc}
0 & \sqrt{N} G_{\alpha} \\
-\sqrt{N} G_{\alpha}^{\dagger} & 0
\end{array}\right),
$$


where we raise and lower indices as $G_{\alpha}=\epsilon_{\alpha \beta} G^{\beta}$, with $\epsilon=i \tilde{\sigma}_{2}=-i \sigma_{2}$. Then the $\mathrm{SU}(2)$ algebra together with the relation $(2.5)$ and the definition of $J_{i}, \bar{J}_{i}$ result in the following (anti)commutation relations

$$
\begin{aligned}
& {\left[\mathbf{J}_{i}, \mathbf{J}_{j}\right]=2 i \epsilon_{i j k} \mathbf{J}_{k}, \quad\left[\mathbf{J}_{i}, \mathbf{J}_{\alpha}\right]=\left(\tilde{\sigma}_{i}\right)_{\alpha \beta} \mathbf{J}^{\beta},} \\
& \left\{\mathbf{J}_{\alpha}, \mathbf{J}_{\beta}\right\}=-\left(\tilde{\sigma}_{i}\right)_{\alpha \beta} \mathbf{J}_{i}=-\left(i \tilde{\sigma}_{2} \tilde{\sigma}_{i}\right)_{\alpha \beta} \mathbf{J}_{i},
\end{aligned}
$$

which is the defining superalgebra $\operatorname{OSp}(1 \mid 2)$ for the fuzzy supersphere of [29].

It is known that the only irreducible representations of $\operatorname{OSp}(1 \mid 2)$ split into the spin- $j$ plus the spin- $\left(j-\frac{1}{2}\right)$ representations of $\mathrm{SU}(2)$, which correspond precisely to the irreducible representation for the $J_{i}(\operatorname{spin} j)$ and $\bar{J}_{i}(\operatorname{spin} j-1 / 2)$ that we are considering here ${ }^{6}$.

As a result, the most general representations of the fuzzy superalgebra, including $G^{\alpha}$ besides $J_{i}, \bar{J}_{i}$, coincide with the most general representations of the two copies of $\mathrm{SU}(2)$. This points to the fact that perhaps the representations in terms of $G^{\alpha}$ are equivalent to the representations of $\mathrm{SU}(2)$. Next we will see that this is indeed the case.

\section{Equivalence of fuzzy sphere constructions}

We now prove that our new definition of the fuzzy 2-sphere in terms of bifundamentals is equivalent to the usual definition in terms of adjoint representations of the $\mathrm{SU}(2)$ algebra.

The ABJM bifundamental scalars are interpreted as Matrix Theory $(N \times N)$ versions of Euclidean coordinates. Accordingly, for our fuzzy space solution in the large $N$-limit one writes $G^{\alpha} \rightarrow \sqrt{N} g^{\alpha}$, with $g^{\alpha}$ some commuting classical objects, to be identified and better understood in due course. In that limit, and similarly writing $J_{i} \rightarrow N x_{i}, \bar{J}_{i} \rightarrow N \bar{x}_{i}$, one has from Sections 2.1.1 and 2.1.2 that the coordinates

$$
x_{i}=\left(\tilde{\sigma}_{i}\right)^{\alpha}{ }_{\beta} g^{\beta} g_{\alpha}^{*}, \quad \bar{x}_{i}=\left(\tilde{\sigma}_{i}\right)^{\alpha}{ }_{\beta} g_{\alpha}^{*} g^{\beta}
$$

are two versions of the same Euclidean coordinate on the 2-sphere, $x_{i} \simeq \bar{x}_{i}$.

In the above construction the 2 -sphere coordinates $x_{i}, \bar{x}_{i}$ are invariant under multiplication of the classical objects $g^{\alpha}$ by a U(1) phase, thus we can define objects $\tilde{g}^{\alpha}$ modulo such a phase, i.e. $g^{\alpha}=e^{i \alpha(\vec{x})} \tilde{g}^{\alpha}$. The GRVV matrices (2.1), that from now on we will denote by $\tilde{G}^{\alpha}$ instead of $G^{\alpha}$, are fuzzy versions of representatives of $\tilde{g}^{\alpha}$, chosen such that $\tilde{g}^{1}=\tilde{g}_{1}^{\dagger}$ (one could of course have chosen a different representative for $\tilde{g}^{\alpha}$ such that $\tilde{g}^{2}=\tilde{g}_{2}^{\dagger}$ instead).

In terms of the $g^{\alpha}$, equation (3.1) is the usual Hopf map from the 3 -sphere $g^{\alpha} g_{\alpha}^{\dagger}=1$ onto the 2 -sphere $x_{i} x_{i}=1$, as we will further discuss in the next section. In this picture, the phase is simply the coordinate on the U(1) fibre of the Hopf fibration, while the $\tilde{g}^{\alpha}$ 's are coordinates on the $S^{2}$ base. While $g^{\alpha}$ are complex coordinates acted upon by $\mathrm{SU}(2)$, the $\tilde{g}^{\alpha}$ are real objects acted upon by the spinor representation of $\mathrm{SO}(2)$, so they can be thought of as Lorentz spinors in two dimensions, i.e. spinors on the 2 -sphere.

The fuzzy version of the full Hopf map, $J_{i}=\left(\tilde{\sigma}_{i}\right)^{\alpha}{ }_{\beta} G^{\beta} G_{\alpha}^{\dagger}$, can be given either using $G^{\alpha}=$ $U \tilde{G}^{\alpha}$ or $G^{\alpha}=\tilde{\hat{G}}^{\alpha} \hat{U}$. The $U$ and $\hat{U}$ are unitary matrices that can themselves be expanded in terms of fuzzy spherical harmonics

$$
U=\sum_{l m} U_{l m} Y_{l m}\left(J_{i}\right)
$$

with $U U^{\dagger}=\hat{U} \hat{U}^{\dagger}=1$, implying that in the large- $N$ limit $(U, \hat{U}) \rightarrow e^{i \alpha(\vec{x})}$.

\footnotetext{
${ }^{6}$ See for instance Appendix $\mathrm{C}$ of [28]. The general spin- $j$ is the $J_{i}$ representation constructed from the GRVV matrices, while the general spin $j-\frac{1}{2}$ is the $\bar{J}_{i}$ representation constructed from the GRVV matrices.
} 
That means that by extracting a unitary matrix from the left or the right of $G^{\alpha}$, i.e. modulo a unitary matrix, the resulting algebra for $\tilde{G}^{\alpha}$

$$
-\tilde{G}^{\alpha}=\tilde{G}^{\beta} \tilde{G}_{\beta}^{\dagger} \tilde{G}^{\alpha}-\tilde{G}^{\alpha} \tilde{G}_{\beta}^{\dagger} \tilde{G}^{\beta}
$$

should then be exactly equivalent to the usual $\mathrm{SU}(2)$ algebra that appears in the adjoint construction: Both should give the same description of the fuzzy 2-sphere. We would next like to prove this equivalence for all possible representations.

\subsection{Representations}

We first note that the irreducible representations of the algebra (3.2), given by the matrices (2.1), indeed give the most general irreducible representations of $\mathrm{SU}(2)$. Defining $J_{ \pm}=J_{1} \pm i J_{2}$, $\bar{J}_{ \pm}=\bar{J}_{1} \pm i \bar{J}_{2}$, we obtain from (2.1) that

$$
\begin{aligned}
& \left(J_{+}\right)_{m, m-1}=2 \sqrt{(m-1)(N-m+1)}=2 \alpha_{\frac{N-1}{2}, m-\frac{N+1}{2}}, \\
& \left(J_{-}\right)_{n-1, n}=2 \sqrt{(n-1)(N-n+1)}=2 \alpha_{\frac{N-1}{2}, n-\frac{N+1}{2},} \\
& \left(J_{3}\right)_{m n}=2\left(m-\frac{N+1}{2}\right) \delta_{m n}
\end{aligned}
$$

and

$$
\begin{aligned}
& \left(\bar{J}_{+}\right)_{m, m-1}=2 \sqrt{(m-2)(N-m+1)}=2 \alpha_{\frac{N-2}{2}, m-\frac{N+2}{2}}, \\
& \left(\bar{J}_{-}\right)_{n-1, n}=2 \sqrt{(n-2)(N-n+1)}=2 \alpha_{\frac{N-2}{2}, n-\frac{N+2}{2}}, \\
& \left(\bar{J}_{3}\right)_{m n}=2\left(m-\frac{N+2}{2}\right) \delta_{m n}+N \delta_{m 1} \delta_{n 1},
\end{aligned}
$$

whereas the general spin- $j$ representation of $\mathrm{SU}(2)$ is

$$
\left(J_{+}\right)_{m, m-1}=\alpha_{j, m}, \quad\left(J_{-}\right)_{n-1, n}=\alpha_{j, n}, \quad\left(J_{3}\right)_{m n}=m \delta_{m n}
$$

(and the rest zero), where

$$
\alpha_{j m} \equiv \sqrt{(j+m)(j-m+1)}
$$

and $m \in-j, \ldots,+j$ takes $2 j+1$ values. Thus the representation for $J_{i}$ is indeed the most general $N=2 j+1$ dimensional representation, and since $\left(\bar{J}_{+}\right)_{11}=\left(\bar{J}_{-}\right)_{11}=\left(\bar{J}_{3}\right)_{11}=0$, the representation for $\bar{J}_{i}$ is also the most general $(N-1)=2\left(j-\frac{1}{2}\right)+1$ dimensional representation.

We still have the $\mathrm{U}(1)$ generators completing the $\mathrm{U}(2)$ symmetry, which in the case of the irreducible GRVV matrices $\tilde{G}^{\alpha}$ are diagonal and give the fuzzy sphere constraint $\tilde{G}^{\alpha} \tilde{G}_{\alpha}^{\dagger} \propto 1$, $\tilde{G}_{\alpha}^{\dagger} \tilde{G}^{\alpha} \propto 1$

$$
J=J_{1}^{1}+J_{2}^{2}=(N-1) \delta_{m n}, \quad \bar{J}=\bar{J}_{1}{ }^{1}+\bar{J}_{2}{ }^{2}=N \delta_{m n}-N \delta_{m 1} \delta_{n 1},
$$

where again $(\bar{J})_{11}=0$, since $\bar{J}_{i}$ is in the $N-1 \times N-1$ dimensional representation: The element $E_{11}=\delta_{m 1} \delta_{n 1}$ is a special operator, so the first element of the vector space on which it acts is also special, i.e. $\mathbf{V}^{-}=V_{N-1}^{-} \oplus V_{1}^{-}$.

Moving to reducible representations of $\mathrm{SU}(2)$, the Casimir operator $\vec{J}^{2}=J_{i} J_{i}$ giving the fuzzy sphere constraint is diagonal, with blocks proportional to the identity. The analogous object that gives the fuzzy sphere constraint in our construction is the operator $J=G^{\alpha} G_{\alpha}^{\dagger}$. 
Indeed, in the case of reducible matrices modulo unitary transformations, $\tilde{G}^{\alpha}$, we find (in the same way as for $\vec{J}^{2}=J_{i} J_{i}$ for the $\mathrm{SU}(2)$ algebra)

$$
J=\operatorname{diag}\left(\left(N_{1}-1\right) \mathbb{1}_{N_{1} \times N_{1}},\left(N_{2}-1\right) \mathbb{1}_{N_{2} \times N_{2}}, \ldots\right)
$$

and similarly for $\bar{J}=G_{\alpha}^{\dagger} G^{\alpha}$

$$
\bar{J}=\operatorname{diag}\left(N_{1}\left(1-E_{11}^{(1)}\right) \mathbb{1}_{N_{1} \times N_{1}}, N_{2}\left(1-E_{11}^{(2)}\right) \mathbb{1}_{N_{2} \times N_{2}}, \ldots\right) .
$$

\subsection{GRVV algebra $\rightarrow \mathrm{SU}(2)$ algebra}

For this direction of the implementation one does not need to consider the particular representations of the algebra; the matrices $\tilde{G}^{\alpha}$ will be kept as arbitrary solutions. We define as before, but now for an arbitrary solution $G^{\alpha}$,

$$
G^{\alpha} G_{\beta}^{\dagger} \equiv J_{\beta}^{\alpha} \equiv \frac{J_{i}\left(\tilde{\sigma}_{i}\right)^{\alpha}{ }_{\beta}+J \delta_{\beta}^{\alpha}}{2} .
$$

Using the GRVV algebra it is straightforward to verify that $G^{\alpha} G_{\alpha}^{\dagger} \equiv J$ commutes with $J_{k}$. Multiplying (3.2) from the right by $\left(\tilde{\sigma}_{k}\right)^{\gamma}{ }_{\alpha} G_{\gamma}^{\dagger}$, one obtains

$$
-J_{k}=G^{\beta} G_{\beta}^{\dagger} J_{k}-J^{\alpha}{ }_{\beta} J_{\gamma}^{\beta}\left(\tilde{\sigma}_{k}\right)_{\alpha}^{\gamma} .
$$

Using the definition for the $J^{\alpha}{ }_{\beta}$ factors in (3.5) and the relation $\left[J, J_{k}\right]=0$, one arrives at

$$
-J_{k}=\frac{i}{2} \epsilon_{i j k} J_{i} J_{j}
$$

which is just the usual $\mathrm{SU}(2)$ algebra.

It is also possible to define

$$
G_{\alpha}^{\dagger} G^{\beta} \equiv \bar{J}_{\alpha}{ }^{\beta} \equiv \frac{\bar{J}_{i}\left(\tilde{\sigma}_{i}\right)^{\beta}{ }_{\alpha}+\bar{J} \delta_{\alpha}^{\beta}}{2}
$$

and similarly obtain $\left[\bar{J}, \bar{J}_{k}\right]=0$. By multiplying (3.2) from the left by $\left(\tilde{\sigma}_{k}\right)^{\gamma}{ }_{\alpha} G_{\gamma}^{\dagger}$, we get in a similar way

$$
-\bar{J}_{k}=\frac{i}{2} \epsilon_{i j k} \bar{J}_{i} \bar{J}_{j}
$$

Thus the general SU(2) algebras for $J_{i}$ and $\bar{J}_{i}$ indeed follow immediately from (3.2) without restricting to the irreducible GRVV matrices.

\section{3 $\quad \mathrm{SU}(2)$ algebra $\rightarrow$ GRVV algebra}

This direction of the implementation is a priori more problematic since, as we have already seen, the representations of $J_{i}$ and $\bar{J}_{i}$ are not independent. For the irreducible case in particular, $V_{N}^{+}$ is replaced by the representation $V_{N-1}^{-} \oplus V_{1}^{-}$, so we need to generalise this identification to reducible representations in order to prove our result. As we will obtain this relation at the end of this section and it should have been the starting point of the proof, we will close with some comments summarising the complete logic.

We will first try to understand the classical limit. The Hopf fibration (3.1) can be rewritten, together with the normalisation condition, as

$$
g^{\alpha} g_{\beta}^{*}=\frac{1}{2}\left[x_{i}\left(\tilde{\sigma}_{i}\right)_{\beta}^{\alpha}+\delta_{\beta}^{\alpha}\right] .
$$


By extracting a phase out of $g^{\alpha}$, we should obtain the variables $\tilde{g}^{\alpha}$ on $S^{2}$ instead of $S^{3}$. Indeed, the above equations can be solved for $g^{\alpha}$ by

$$
g^{\alpha}=\left(\begin{array}{c}
g^{1} \\
g^{2}
\end{array}\right)=\frac{e^{i \phi}}{\sqrt{2\left(1+x_{3}\right)}}\left(\begin{array}{c}
1+x_{3} \\
x_{1}-i x_{2}
\end{array}\right)=e^{i \phi} \tilde{g}^{\alpha},
$$

where $e^{i \phi}$ is an arbitrary phase.

In the fuzzy case $G^{\alpha}$ and $G_{\beta}^{\dagger}$ do not commute, and there are two different kinds of equations corresponding to $J_{i}$ and $\bar{J}_{i}$,

$$
G^{\alpha} G_{\beta}^{\dagger} \equiv \frac{1}{2}\left[J_{i}\left(\tilde{\sigma}_{i}\right)_{\beta}^{\alpha}+\delta_{\beta}^{\alpha} J\right], \quad G_{\beta}^{\dagger} G^{\alpha} \equiv \frac{1}{2}\left[\bar{J}_{i}\left(\tilde{\sigma}_{i}\right)_{\beta}^{\alpha}+\delta_{\beta}^{\alpha} J\right] .
$$

We also impose that $\left[J, J_{k}\right]=0,\left[\bar{J}, \bar{J}_{k}\right]=0$, so that $J$ and $\bar{J}$ are diagonal and proportional to the identity in the irreducible components of $J_{i}$.

We solve the first set of equations in (3.7) by writing $G^{1} G_{1}^{\dagger}=\frac{1}{2}\left(J+J_{3}\right)$, for which the most general solution is $G_{1}=T U$, with $T$ a Hermitian and $U$ a unitary matrix. Since $J+J_{3}$ is real and diagonal, by defining

$$
T=\frac{1}{\sqrt{2}}\left(J+J_{3}\right)^{1 / 2}
$$

we obtain

$$
G^{\alpha}=\left(\begin{array}{c}
G^{1} \\
G^{2}
\end{array}\right)=\left(\begin{array}{c}
J+J_{3} \\
J_{1}-i J_{2}
\end{array}\right) \frac{T^{-1}}{2} U_{N \times N}=\tilde{G}^{\alpha} U_{N \times N} .
$$

Thus $\tilde{G}^{\alpha}$ is also completely determined by $J_{i}, J$.

Similarly, the second set of equations in (3.7) can be solved by considering $G_{1}^{\dagger} G^{1}=\frac{1}{2}\left(\bar{J}+\bar{J}_{3}\right)$, for which the most general solution is $G^{1}=\hat{U} \tilde{T}$, where as before

$$
\tilde{T}=\frac{1}{\sqrt{2}}\left(\bar{J}+\bar{J}_{3}\right)^{1 / 2},
$$

to obtain

$$
G^{\alpha}=\left(\begin{array}{c}
G^{1} \\
G^{2}
\end{array}\right)=\hat{U}_{\bar{N} \times \bar{N}} \frac{\tilde{T}^{-1}}{2}\left(\begin{array}{c}
\bar{J}+\bar{J}_{3} \\
\bar{J}_{1}-i \bar{J}_{2}
\end{array}\right)=\hat{U} \tilde{\hat{G}}^{\alpha}
$$

Thus $\tilde{\hat{G}}^{\alpha}$ is completely determined by $\bar{J}_{i}, \bar{J}$.

Comparing the two formulae for $G^{\alpha}$ we see that they are compatible if and only if

$$
\hat{U}=T U \tilde{T}^{-1} \quad \text { and } \quad \bar{J}_{1}-i \bar{J}_{2}=\tilde{T}^{2} U^{-1} T^{-1}\left(J_{1}-i J_{2}\right) T^{-1} U,
$$

where $U$ is an arbitrary unitary matrix. These equations define an identification between the two representations of SU(2), in terms of $J_{i}$ and $\bar{J}_{i}$, needed in order to establish the equivalence with the GRVV matrices.

We now analyse the equivalence for specific representations. For the irreducible representations of $\mathrm{SU}(2)$, we define $\bar{J}_{i}$ from $J_{i}$ as before $\left(V_{N}^{+} \rightarrow V_{N-1}^{-} \oplus V_{1}^{-}\right)$and $J=(N-1) \mathbb{1}_{N \times N}$, $\bar{J}=N\left(1-E_{11}\right) \mathbb{1}_{N \times N}$. For reducible representations of $\mathrm{SU}(2), J_{i}$ can be split such that $J_{3}$ is block-diagonal, with various irreps added on the diagonal. One must then take $J$ and $\bar{J}$ of the form in (3.3) and (3.4). The condition (3.10) is solved by $U=1$ and $J_{1}, J_{2}$ block diagonal, with the blocks being the irreps of dimensions $N_{1}, N_{2}, N_{3}, \ldots$, and the $\bar{J}_{1}, \bar{J}_{2}$ being also block diagonal, but where each $N_{k} \times N_{k}$ irrep block is replaced with the $\left(N_{k}-1\right) \times\left(N_{k}-1\right)$ irrep block, plus an $E_{11}^{(k)}$, just as for the GRVV matrices.

We can hence summarise the proof a posteriori in the following steps: 
1. Start with $J_{i}(i=1,2,3)$ in the reducible representation of $\mathrm{SU}(2)$, i.e. block diagonal with the blocks being irreps of dimensions $N_{1}, N_{2}, N_{3}, \ldots$.

2. Take $J=G^{\alpha} G_{\alpha}^{\dagger}$ and $\bar{J}=G_{\alpha}^{\dagger} G^{\alpha}$ as in (3.3) and (3.4) since these are necessary conditions for the $G^{\alpha}$ to satisfy the GRVV algebra. The condition $\left[J, J_{k}\right]=0$ is used here.

3. The $\bar{J}_{i}$ are completely determined (up to conventions) from $J_{i}, J$ and $\bar{J}$ by (3.10) and the condition $\left[\bar{J}, \bar{J}_{k}\right]=0$.

4. The $\tilde{G}^{\alpha}$ are then uniquely determined by (3.8), while the $\tilde{\hat{G}}^{\alpha}$ by (3.9).

5. The $\tilde{G}^{\alpha}$ and $\tilde{\hat{G}}^{\alpha}$ defined as above indeed satisfy the GRVV algebra.

\section{$4 \quad$ Fuzzy Hopf fibration and fuzzy Killing spinors}

Having established the equivalence between the adjoint (usual) and the bifundamental (in terms of $\tilde{G}^{\alpha}$ ) formulations of the fuzzy $S^{2}$ we turn towards ascribing an interpretation to the matrices $\tilde{G}^{\alpha}$ themselves.

\subsection{Hopf fibration interpretation}

One such interpretation was alluded to already in (2.2), where the fuzzy (matrix) coordinates $G^{\alpha}$ were treated as complex spacetime coordinates. The irreducible GRVV matrices satisfy $\tilde{G}^{1} \tilde{G}_{1}^{\dagger}+\tilde{G}^{2} \tilde{G}_{2}^{\dagger}=N-1$ and $\tilde{G}^{1}=\tilde{G}_{1}^{\dagger}$. The first relation suggests a fuzzy 3 -sphere, but the second is an extra constraint which reduces the geometry to a $2 \mathrm{~d}$ one. This is in agreement with the fuzzy $S^{2}$ equivalence that we already established in the previous section. The matrices $\tilde{G}^{\alpha}$ are viewed as representatives when modding out the $\mathrm{U}(N)$ symmetry, and the condition $\tilde{G}^{1}=\tilde{G}_{1}^{\dagger}$ amounts to a choice of representative of the equivalence class.

The construction of the fuzzy $S^{2}$ in usual (Euclidean) coordinates was obtained by

$$
\begin{aligned}
& J_{i}=\left(\tilde{\sigma}_{i}\right)_{\beta}^{\alpha} G^{\beta} G_{\alpha}^{\dagger}, \\
& x_{i}=\frac{J_{i}}{\sqrt{N^{2}-1}} \Rightarrow\left\{\begin{array}{l}
x_{1}=\frac{J_{1}}{\sqrt{N^{2}-1}}=\frac{1}{\sqrt{N^{2}-1}}\left(G^{1} G_{2}^{\dagger}+G^{2} G_{1}^{\dagger}\right), \\
x_{2}=\frac{J_{2}}{\sqrt{N^{2}-1}}=\frac{i}{\sqrt{N^{2}-1}}\left(G^{1} G_{2}^{\dagger}-G^{2} G_{1}^{\dagger}\right), \\
x_{3}=\frac{J_{3}}{\sqrt{N^{2}-1}}=\frac{1}{\sqrt{N^{2}-1}}\left(G^{1} G_{1}^{\dagger}-G^{2} G_{2}^{\dagger}\right),
\end{array}\right. \\
& \frac{G^{\alpha}}{\sqrt{N}} \rightarrow g^{\alpha}
\end{aligned}
$$

and we already stated that the relation between $g^{\alpha}$ and $x_{i}$ is the classical Hopf map $S^{3} \stackrel{\pi}{\rightarrow}$ $S^{2},(3.1)$.

Indeed, the description of the Hopf map in classical geometry is given as follows: One starts with Cartesian coordinates $X_{1}, X_{2}, X_{3}, X_{4}$ on the unit $S^{3}$ with

$$
X_{1}^{2}+X_{2}^{2}+X_{3}^{2}+X_{4}^{2}=1
$$

and then goes to complex variables $Z^{1}=X_{1}+i X_{2}, Z^{2}=X_{3}+i X_{4}$, satisfying $Z^{\alpha} Z_{\alpha}^{*}=1$. The Hopf map defines Cartesian coordinates on the unit $S^{2}$ base of the fibration by

$$
x_{i}=\left(\tilde{\sigma}_{i}\right)_{\beta}^{\alpha} Z^{\beta} Z_{\alpha}^{*}
$$


which is invariant under an $S^{1}$ fibre defined by multiplication of $Z^{\alpha}$ by a phase. The $x_{i}$ are Euclidean coordinates on an $S^{2}$ since

$$
x_{i} x_{i}=\left(\tilde{\sigma}_{i}\right)_{\beta}^{\alpha}\left(\tilde{\sigma}_{i}\right)_{\nu}^{\mu} Z^{\beta} Z_{\alpha}^{*} Z^{\nu} Z_{\mu}^{*}=1
$$

and this identifies $Z^{\alpha} \equiv g^{\alpha}$ from above.

Let us now work in the opposite direction, starting from the classical limit and discretising the geometry by demoting the Hopf map (4.1) from classical coordinates to finite matrices. We need matrices for $Z^{\alpha}$ which we call $G^{\alpha}$. The coordinates $x_{i}$ transform in the spin-1 representation of $\mathrm{SU}(2)$. If we want to build them from bilinears of the form $G^{\dagger} G$ we need $G, G^{\dagger}$ to transform in the spin- $\frac{1}{2}$ representation. We also want a gauge symmetry to extend the $\mathrm{U}(1)$ invariance of $Z^{\alpha}$ (the $S^{1}$ fiber of the Hopf map), and for $N$-dimensional matrices $\mathrm{U}(N)$ is the desired complex gauge invariance that plays that role.

In the usual fuzzy 2 -sphere, the $x_{i}$ are operators mapping an irreducible $N$-dimensional SU(2) representation $V_{N}$ to itself. It is possible to do this in an $\mathrm{SU}(2)$-covariant fashion because the tensor product of spin-1 with $V_{N}$ contains $V_{N}$. Since $G^{\alpha}$ are spin- $\frac{1}{2}$, and $\frac{1}{2} \otimes V_{N}=V_{N+1} \oplus V_{N-1}$ does not contain $V_{N}$, we need to work with reducible representations in order to have $G^{\alpha}$ map the representation back to itself. The simplest thing to do would be to consider the representation $V_{N} \oplus V_{N-1}$. The next simplest thing is to work with $V_{N} \oplus\left(V_{N-1} \oplus V_{1}\right)$ and this possibility is chosen by the GRVV matrices [18] and allows a gauge group $\mathrm{U}(N) \times \mathrm{U}(\bar{N})$ which has a $\mathbb{Z}_{2}$ symmetry of exchange needed to preserve parity.

So the unusual property of the GRVV matrices $\tilde{G}$, the difference between $\mathbf{V}^{+}=V_{N}$ and $\mathbf{V}^{-}=V_{N-1} \oplus V_{1}$ follows from requiring a matrix realisation of the fuzzy $S^{2}$ base of the Hopf fibration. These in turn lead to the $\mathrm{SU}(2)$ decompositions of $\operatorname{End}\left(\mathbf{V}^{+}\right), \operatorname{End}\left(\mathbf{V}^{-}\right)$, $\operatorname{Hom}\left(\mathbf{V}^{+}, \mathbf{V}^{-}\right), \operatorname{Hom}\left(\mathbf{V}^{-}, \mathbf{V}^{+}\right)$, for the fluctuation matrices that we saw in Section 2.2. ${ }^{7}$

The $x_{i}, G, G^{\dagger}$ are operators in $\mathbf{V}^{+} \oplus \mathbf{V}^{-}$which is isomorphic, as a vector space, to $\mathbf{V}_{N} \otimes V_{2}$. The endomorphisms of $\mathbf{V}_{N}$ correspond to the fuzzy sphere. The $N$ states of $\mathbf{V}_{N}$ generalise the notion of points on $S^{2}$ to noncommutative geometry. The 2-dimensional space $V_{2}$ is invariant under the $\mathrm{SU}(2)$. It is acted on by $G, G^{\dagger}$ which have charge $+1,-1$ under the $\mathrm{U}(1)$ (corresponding to $(J, \bar{J}))$ acting on the fibre of the Hopf fibration, so we also have two points on top of our fuzzy $S^{2}$.

Since in this subsection we looked at a fibration of $S^{3}$, we need to emphasise that the fluctuation analysis does not have enough modes to describe the full space of functions on $S^{3}$, even if we drop the requirement of $\mathrm{SO}(4)$ covariance and allow for the possibility of an $\mathrm{SU}(2) \times$ $\mathrm{U}(1)$ description. As we explained above, the only remnant of the circle in the matrix construction is the multiplicity associated with having states $|+\rangle,|-\rangle$ in $\mathbf{V}^{+}$and $\mathbf{V}^{-}$. A classical description of the $S^{3}$ metric as a Hopf fibration contains a coordinate $y$ transverse to the $S^{2}$. Instead, the matrix fluctuations of our solution are mapped to functions on $S^{2}$ and hence lead to a field theory on $S^{2}$.

\subsection{Killing spinor interpretation}

We will close this circle of arguments by interpreting the classical objects $\tilde{g}^{\alpha}$, obtained in the large- $N$ limit of $\tilde{G}^{\alpha}$, as Killing spinors and fuzzy Killing spinors on the 2-sphere respectively.

We have seen that in the classical limit the relation between $J_{i}$ and $G^{\alpha}$ becomes the first Hopf map (3.1), and hence can be thought of as its fuzzy version. However, the above Hopf relation is invariant under multiplication by an arbitrary phase corresponding to shifts on the $S^{1}$ fibre,

\footnotetext{
${ }^{7}$ The usual fuzzy $S^{2}$ has also been discussed in terms of the Hopf fibration, where the realisation of the SU(2) generators in terms of bilinears in Heisenberg algebra oscillators yields an infinite dimensional space which admits various projections to finite $N$ constructions [30]. In that case the $x_{i}$ are not bilinears in finite matrices.
} 
so the objects $\tilde{g}^{\alpha}$ obtained by extracting that phase in (3.6), i.e.

$$
\tilde{g}^{\alpha}=\frac{1}{\sqrt{2\left(1+x_{3}\right)}}\left(\begin{array}{c}
1+x_{3} \\
x_{1}-i x_{2}
\end{array}\right)
$$

are instead defined on the classical $S^{2}$. In the Hopf fibration, the index of $g^{\alpha}$ is a spinor index of the global SO(3) symmetry for the 2-sphere. By extracting the $S^{1}$ phase one obtains a real (or rather, subject to a reality condition) $\tilde{g}^{\alpha}$ and the $\alpha$ can be thought of as describing a (Majorana) spinor of the $\mathrm{SO}(2)$ local Lorentz invariance on the 2 -sphere. We will argue that the latter is related to a Killing spinor. Note that this type of index identification easily extends to all even spheres.

In the fuzzy version of (4.2), the $\tilde{G}^{\alpha}$ obtained from $G^{\alpha}$ by extracting a unitary matrix, are real objects defined on the fuzzy $S^{2}$. They equal the GRVV matrices in the case of irreducible representations, or

$$
\tilde{G}=\left(\begin{array}{c}
J+J_{3} \\
J_{1}-i J_{2}
\end{array}\right) \frac{T^{-1}}{2}
$$

in general.

The standard interpretation, inherited from the examples of the $\mathrm{SU}(2)$ fuzzy 2 -sphere and other spaces, is that the matrix indices give rise to the dependence on the sphere coordinates and the index $\alpha$ is a global symmetry index. However, we have just seen that already in the classical picture one can identify the global symmetry spinor index with the local Lorentz spinor index. Therefore we argue that the correct interpretation of the classical limit for $\tilde{G}^{\alpha}$ is as a spinor with both global and local Lorentz indices, i.e. the Killing spinors on the sphere $\eta^{\alpha I}$. In the following we will use the index $\alpha$ interchangeably for the two.

In order to facilitate the comparison with the Killing spinors, we express the classical limit of the $J_{i}-\tilde{G}^{\alpha}$ relation as

$$
x_{i} \simeq \bar{x}_{i}=\left(\sigma_{i}\right)_{\alpha}^{\beta} \tilde{g}_{\beta}^{\dagger} \tilde{g}^{\alpha} .
$$

\section{Killing spinors on $S^{n}$}

We now review some of the key facts about Killing spinors that we will need for our discussion. For more details, we refer the interested reader to e.g. [31, 32, 33, 34, 35].

On a general sphere $S^{n}$, one has Killing spinors satisfying

$$
D_{\mu} \eta(x)= \pm \frac{i}{2} m \gamma_{\mu} \eta(x) .
$$

There are two kinds of Killing spinors, $\eta^{+}$and $\eta^{-}$, which in even dimensions are related by the chirality matrix, i.e. $\gamma_{n+1}$, through $\eta^{+}=\gamma_{n+1} \eta^{-}$, as can be easily checked. The Killing spinors on $S^{n}$ satisfy orthogonality, completeness and a reality condition. The latter depends on the application, sometimes taken to be the modified Majorana condition, which mixes (or identifies) the local Lorentz spinor index with the global symmetry spinor index of $S^{n}$. For instance, on $S^{4}$ the orthogonality and completeness are respectively ${ }^{8}$,

$$
\bar{\eta}^{I} \eta^{J}=\Omega^{I J} \quad \text { and } \quad \eta_{J}^{\alpha} \bar{\eta}_{\beta}^{J}=-\delta_{\beta}^{\alpha},
$$

\footnotetext{
${ }^{8}$ The charge conjugation matrix in $n$ dimensions satisfies in general

$$
C^{T}=\kappa C, \quad \gamma_{\mu}^{T}=\lambda C \gamma_{\mu} C^{-1},
$$

where $\kappa= \pm, \lambda= \pm$ and it is used to raise/lower indices. The Majorana condition is then given by

$$
\bar{\eta}=\eta^{T} C .
$$
}


where the index $I$ is an index in a spinorial representation of the $\mathrm{SO}(n+1)_{G}$ invariance group of the sphere and the index $\alpha$ is an index in a spinorial representation of the $\mathrm{SO}(n)_{L}$ local Lorentz group on the sphere. The indices are then identified by the modified Majorana spinor condition as follows ${ }^{9}$

$$
\bar{\eta}^{I} \equiv\left(\eta^{I}\right)^{T} C_{-}^{(n)}=-\left(\eta^{J}\right)^{\dagger} \gamma_{n+1} \Omega^{I J}
$$

where $\Omega^{I J}=i \sigma_{2} \otimes \mathbb{1}_{\frac{n}{2} \times \frac{n}{2}}$ is the invariant tensor of $\operatorname{Sp}\left(\frac{n}{2}\right)$, satisfying $\Omega^{I J} \Omega_{J K}=\delta_{K}^{I}$.

The Euclidean coordinates of $S^{n}$ are bilinear in the Killing spinors

$$
x_{i}=\left(\Gamma_{i}\right)_{I J} \bar{\eta}^{I} \gamma_{n+1} \eta^{J},
$$

where $\eta$ are of a single kind (+ or - ), or equivalently $\bar{\eta}_{+}^{I} \eta_{-}^{J}$. In the above the $\Gamma$ are in $\mathrm{SO}(n+1)_{G}$, while the $\gamma$ in $\mathrm{SO}(n)_{L}$.

Starting from Killing spinors on $S^{n}$, one can construct all the higher spherical harmonics. As seen in equation (4.4), Euclidean coordinates on the sphere are spinor bilinears. In turn, symmetric traceless products of the $x_{i}$ 's construct the scalar spherical harmonics $Y^{k}\left(x_{i}\right) \cdot{ }^{10}$ One can also construct the set of spinorial spherical harmonics by acting with an appropriate operator on $Y^{k} \eta^{I}$

$$
\begin{aligned}
& \Xi^{k,+}=\left[(k+n-1+i \not D) Y^{k}\right] \eta_{+}, \\
& \Xi^{k,-}=\left[(k+n-1+i \not D) Y^{k}\right] \eta_{-}=\left[(k+1+i \not D) Y^{k+1}\right] \eta_{+} .
\end{aligned}
$$

Note that in the above the derivatives act only on the scalar harmonics $Y^{k}$.

Any spinor on the sphere can be expanded in terms of spinorial spherical harmonics, $\Psi=$ $\sum_{k} \psi_{k} \Xi^{k, \pm}$. Consistency imposes that the $\Xi^{k, \pm}$ can only be commuting spinors. The Killing spinors are then themselves commuting spinors, as they are used to construct the spinorial spherical harmonics.

For higher harmonics the construction extends in a similar way but the formulae are more complicated and, as we will not need them for our discussion, we will not present them here. The interested reader can consult e.g. [37].

\section{Killing spinors on $S^{2}$ and relation between spinors}

For the particular case of the $S^{2}, \gamma_{i}=\Gamma_{i}=\sigma_{i}$ for both the $\mathrm{SO}(2)_{L}$ and the $\mathrm{SO}(3)_{G}$ Clifford algebras. Then the two $C$-matrices can be chosen to be: $C_{+}=-\sigma_{1}$, giving $\kappa=\lambda=+$, and $C_{-}=i \sigma_{2}=\epsilon$, giving $\kappa=\lambda=-$. Note that with these conventions one has $C_{-} \gamma_{3}=i \sigma_{2} \sigma_{3}=$ $-\sigma_{1}=C_{+}$. In the following we will choose the Majorana condition to be defined with respect to $C_{-}$.

Equation (4.4) then gives for $n=2$

$$
\bar{\eta}^{I}=\left(\eta^{T}\right)^{I} C_{-} \Rightarrow x_{i}=\left(\sigma_{i}\right)_{I J}\left(\eta^{T}\right)^{I} C_{-} \gamma_{3} \eta^{J} .
$$

The orthonormality and completeness conditions for the Killing spinors on $S^{2}$ are

$$
\bar{\eta}^{I} \eta^{J}=\epsilon^{I J} \quad \text { and } \quad \eta_{J}^{\alpha} \bar{\eta}_{\beta}^{J}=-\delta_{\beta}^{\alpha},
$$

while the modified Majorana condition is

$$
\left(\eta^{J}\right)^{\dagger}=\epsilon_{I J} \bar{\eta}^{I} \equiv \epsilon_{I J}\left(\eta^{I}\right)^{T} C_{-} .
$$

\footnotetext{
${ }^{9}$ For more details on Majorana spinors and charge conjugation matrices see [31, 36] and the Appendix of [35].

${ }^{10}$ These are the higher dimensional extensions of the usual spherical harmonics $Y^{l m}\left(x_{i}\right)$ for $S^{2}$.
} 
Since $C_{-}=\epsilon$, by making both indices explicit and by renaming the index $I$ as $\dot{\alpha}$ for later use, one also has

$$
\left(\eta^{\alpha \dot{\alpha}}\right)^{\dagger}=\eta_{\alpha \dot{\alpha}} \equiv \epsilon_{\alpha \beta} \epsilon_{\dot{\alpha} \dot{\beta}} \eta^{\beta \dot{\beta}}
$$

Finally, the spinorial spherical harmonics on $S^{2}$ are

$$
\Xi_{l m}^{ \pm}=\left[(l+1+i \not D) Y_{l m}\right] \eta_{ \pm}
$$

and thus the spherical harmonic expansion of an $S^{2}$-fermion is (writing explicitly the sphere fermionic index $\alpha$ )

$$
\psi^{\alpha}=\sum_{l m, \pm} \psi_{l m, \pm} \Xi_{l m}^{ \pm, \alpha}=\sum_{l m, \pm}\left[\psi_{l m, \pm}(l+1+i \not D) Y_{l m}\right]_{\beta}^{\alpha} \eta_{ \pm}^{\beta} .
$$

To construct explicitly the Killing spinor, we must first define a matrix $S$, that can be used to relate between the two different kinds of spinors on $S^{2}$, spherical and Euclidean.

On the 2-sphere, one defines the Killing vectors $K_{i}^{a}$ such that the adjoint action of the $\mathrm{SU}(2)$ generators on the fuzzy sphere fields becomes a derivation in the large- $N$ limit ${ }^{11}$

$$
\left[J_{i}, \cdot\right] \rightarrow 2 i K_{i}^{a} \partial_{a}=2 i \epsilon_{i j k} x_{j} \partial_{k} .
$$

One can then explicitly check that $K_{i}^{a}$ produces a Lorentz transformation on the gamma matri$\operatorname{ces}^{12}$

$$
K_{i}^{a}\left(\tilde{\sigma}_{i}\right)^{\alpha}{ }_{\beta}=-e^{a m}\left(S \sigma^{m} S^{-1}\right)_{\beta}{ }^{\alpha} \equiv-\left(S \gamma^{a} S^{-1}\right)_{\beta}{ }^{\alpha},
$$

where $e^{a m}$ is the vielbein on the sphere and $S$ is a unitary matrix defining the transformation $(|a|=1)$

$$
S=a\left(\begin{array}{cc}
-\sin \frac{\theta}{2} e^{i \phi / 2} & -i \cos \frac{\theta}{2} e^{i \phi / 2} \\
\cos \frac{\theta}{2} e^{-i \phi / 2} & -i \sin \frac{\theta}{2} e^{-i \phi / 2}
\end{array}\right) .
$$

Imposing the (symplectic) reality condition on $S$

$$
\epsilon_{\alpha \beta}\left(S^{-1}\right)_{\gamma}^{\beta} \epsilon^{\gamma \delta}=\left(S^{T}\right)_{\alpha}^{\delta}=S_{\alpha}^{\delta},
$$

we fix $a=\sqrt{i}^{*}$ and obtain the relations

$$
\begin{aligned}
& \left(S \sigma_{i} S^{-1}\right)_{\alpha}^{\beta}=\left(S \sigma_{i} S^{-1}\right)_{\alpha}^{\beta}, \quad\left(S \gamma_{3} S^{-1}\right)_{\beta}^{\alpha}=-x_{i}\left(\tilde{\sigma}_{i}\right)_{\beta}^{\alpha}, \\
& \left(S \gamma_{a} S^{-1}\right)_{\beta}^{\alpha}=-h_{a b} K_{i}^{b}\left(\tilde{\sigma}_{i}\right)^{\alpha}{ }_{\beta} .
\end{aligned}
$$

If one has real spinors obeying

$$
\left(\chi_{\alpha \dot{\alpha}}\right)^{\dagger}=\chi^{\alpha \dot{\alpha}} \equiv \epsilon^{\alpha \beta} \epsilon^{\dot{\alpha} \dot{\beta}} \chi_{\beta \dot{\beta}},
$$

which was identified in (4.6) as the modified Majorana spinor condition, it follows from (4.7) that rotation by the matrix $S$ preserves this relation, i.e.

$$
\left(\left(\chi_{\dot{\alpha}} S\right)_{\alpha}\right)^{\dagger}=\left(S^{-1} \chi^{\dot{\alpha}}\right)^{\alpha} \equiv-\epsilon^{\dot{\alpha} \dot{\beta}}\left(S^{-1}\right)^{\alpha \beta} \chi_{\beta \dot{\beta}}=\epsilon^{\dot{\alpha} \dot{\beta}} \epsilon^{\alpha \beta}\left(\chi_{\dot{\beta}} S\right)_{\beta} .
$$

\footnotetext{
${ }^{11}$ Precise expressions for the Killing vectors as well as a set of useful identities can be found in Appendix A of $[10]$.

${ }^{12} \mathrm{~A}$ Lorentz transformation on the spinors acts as $\Lambda^{\mu}{ }_{\nu} \gamma^{\nu}=S \gamma^{\mu} S^{-1}$, with $S$ unitary.
} 
We can now define the explicit form of the Killing spinor

$$
\eta^{I \alpha}=\left(S^{-1}\right)^{\alpha}{ }_{\beta} \eta_{0}^{I \beta}=\frac{1}{\sqrt{2}}\left(S^{-1}\right)_{\beta}^{\alpha} \epsilon^{\beta I}=\frac{1}{\sqrt{2}} S^{I}{ }_{J} \epsilon^{\alpha J},
$$

where in the last equality we used the (symplectic) reality condition (4.7) on $S$. From (4.9) it is clear that the $\eta^{I \alpha}$ obey the modified Majorana condition. It is then possible to use (4.8) to prove that

$$
x_{i}=\left(\sigma_{i}\right)_{I J} \bar{\eta}^{I} \gamma_{3} \eta^{J}
$$

hence verifying that the $\eta^{I \alpha}$ are indeed Killing spinors. One can also explicitly check that

$$
D_{a}\left(\left(S^{-1}\right)^{\alpha}{ }_{\beta} \epsilon^{\beta I}\right)=+\frac{i}{2}\left(\gamma_{a}\right)_{\beta}^{\alpha}\left(S^{-1}\right)_{\gamma}^{\beta} \epsilon^{\gamma I},
$$

which in turn means that

$$
\frac{1}{\sqrt{2}}\left(S^{-1}\right)_{\beta}^{\alpha} \epsilon^{\beta I}=\eta_{+}^{\alpha I} .
$$

\section{Identification with Killing spinor}

Using (4.6), we rewrite (4.5) as

$$
x_{i}=\left(\sigma_{i}\right)_{J}^{I}\left(\eta^{I}\right)^{\dagger} \gamma_{3} \eta^{J}=\left(\tilde{\sigma}_{i}\right)_{J}^{I}\left(\sqrt{2} P_{+} \eta^{I}\right)^{\dagger}\left(\sqrt{2} P_{+} \eta^{J}\right),
$$

where $P_{ \pm}=\frac{1}{2}\left(1 \pm \gamma_{3}\right)$. Now comparing (4.10) with (4.3) one is led to the following natural large- $N$ relation, $\tilde{G}^{\alpha} \rightarrow \sqrt{2 N} P_{+} \eta^{I}$, provided the spinor indices $\alpha$ and $I$ get identified, i.e.

$$
\frac{\tilde{G}^{\alpha}}{\sqrt{N}} \equiv \tilde{g}^{\alpha} \leftrightarrow \tilde{g}^{I} \equiv \sqrt{2} P_{+} \eta^{I}=\left(P_{+}\right)^{\alpha}{ }_{\beta}\left(S^{-1}\right)_{\gamma}^{\beta} \epsilon^{\gamma I}=\left(P_{+}\right)^{\alpha}{ }_{\beta} S^{I}{ }_{J} \epsilon^{\beta J}=S^{I}{ }_{J}\left(P_{-}\right)^{J}{ }_{K} \epsilon^{\alpha K}
$$

Thus, the Weyl projection can be thought of as 'removing' either $\alpha$ or $I$, since only one of the two spinor components is non-zero.

In order to further check this proposed identification at large- $N$ we now calculate

$$
\partial_{a}\left(\sqrt{2} P_{+} \eta^{I}\right)=-\frac{i}{2}\left(S \gamma_{a} S^{-1}\right)_{J}^{I}\left(\sqrt{2} P_{+} \eta^{J}\right)+\tilde{T}_{a}\left(\sqrt{2} P_{+} \eta^{I}\right),
$$

where $\tilde{T}_{\theta}=0$ and $\tilde{T}_{\phi}=\frac{i}{2} \cos \theta$ and

$$
\left(\partial_{a} S\right) S^{-1}=-\frac{i}{2} S \gamma_{a} S^{-1}+S T_{a} S^{-1}
$$

by explicitly evaluation, with $T_{\theta}=0$ and $T_{\phi}=-\frac{i}{2} \cos \theta \gamma_{3}$.

This needs to be compared with the analogous result given in equation (4.48) of [9] from the classical limit of the adjoint action of $J_{i}$ on $\tilde{G}^{\alpha}$, i.e. from $\left[J_{i}, \tilde{G}^{\alpha}\right]$,

$$
\partial_{a} \tilde{g}^{\alpha}=\frac{i}{2} \hat{h}_{a b} K_{i}^{b}\left(\tilde{\sigma}_{i}\right)^{a}{ }_{\beta} \tilde{g}^{\beta}=-\frac{i}{2}\left(S \gamma_{a} S^{-1}\right)^{\alpha}{ }_{\beta} \tilde{g}^{\beta} .
$$

In [9] it was also verified that the above could reproduce the correct answer for $\partial_{a} x_{i}$, which can be rewritten as

$$
\partial_{a} x_{i}=-\frac{i}{2} \tilde{g}_{\alpha}^{\dagger}\left[\left(\tilde{\sigma}_{i}\right)_{\beta}^{\alpha}\left(S \gamma_{a} S^{-1}\right)_{\gamma}^{\beta}-\left(S \gamma_{a} S^{-1}\right)_{\beta}^{\alpha}\left(\tilde{\sigma}_{i}\right)_{\gamma}^{\beta}\right] \tilde{g}^{\gamma} .
$$


Note that even though there is a difference between (4.11) and (4.12), given by the purely imaginary term $\tilde{T}_{a}$ that is proportional to the identity, the two answers for $\partial_{a} x_{i}$ exactly agree, since in that case the extra contribution cancels. This extra term is a reflection of a double ambiguity: First, the extra index $\alpha$ on $\eta^{I}$ can be acted upon by matrices, even though it is Weylprojected, in effect multiplying the Weyl-projected $\eta^{I}$ by a complex number; if the complex number is a phase, it will not change any expressions where the extra index is contracted, thus we have an ambiguity against multiplication by a phase. Second, $\tilde{g}^{\alpha}$ is just a representative of the reduction of $g^{\alpha}$ by an arbitrary phase, so it is itself only defined up to a phase. The net effect is that the identification of the objects in (4.11) and (4.12) is only up to a phase. Indeed, locally, near $\phi \simeq 0$, one could write

$$
\tilde{g}^{\alpha} e^{\frac{i}{2} \phi \cos \theta} \leftrightarrow \sqrt{2} P_{+} \eta^{I}
$$

but it is not possible to get an explicit expression for the phase over the whole sphere.

\subsection{Generalisations}

On a general $S^{2 n}$ some elements of the above analysis of fuzzy Killing spinors carry through. That is because even though it is possible to write for every $S^{2 n}$

$$
x_{A}=\bar{\eta}^{I}\left(\Gamma_{A}\right)_{I J} \gamma_{2 n+1} \eta^{J},
$$

where $\eta^{I}$ are the Killing spinors, one only has possible fuzzy versions of the quaternionic and octonionic Hopf maps to match it against, i.e. for $2 n=4,8$. We will next find and interpret the latter in terms of Killing spinors on the corresponding spheres.

$S^{4}$

The second Hopf map, $S^{7} \stackrel{\pi}{\rightarrow} S^{4}$, is related to the quaternionic algebra. Expressing the $S^{7}$ in terms of complex coordinates $g^{\alpha}$, now with $\alpha=1, \ldots, 4$, the sphere constraint becomes $g^{\alpha} g_{\alpha}^{\dagger}=1$ $\left(g^{\alpha} g_{\alpha}^{\dagger}=1 \Rightarrow x_{A} x_{A}=1 ; A=1, \ldots, 5\right)$. The map in this case is (see for instance [38])

$$
x_{A}=g^{\beta}\left(\Gamma_{A}\right)^{\alpha}{ }_{\beta} g_{\alpha}^{\dagger},
$$

with $\left(\Gamma_{A}\right)^{\alpha}{ }_{\beta}$ the $4 \times 4 \mathrm{SO}(5)$ gamma matrices ${ }^{13}$. Here we have identified the spinor index $I$ of $\mathrm{SO}(5)$ with the Lorentz spinor index $\alpha$ of $\mathrm{SO}(4)$.

Initially, the $g^{\alpha}$ 's are complex coordinates acted upon by $\mathrm{SU}(4)$, but projecting down to the base of the Hopf fibration we replace $g^{\alpha}$ in the above formula with real $\tilde{g}^{\alpha}$ 's, instead acted upon by the spinorial representation of $\mathrm{SO}(4)$, i.e. by spinors on the 4 -sphere. This process is analogous to what we saw for the case of the 2 -sphere. Once again, it is possible to identify $\tilde{g}^{\alpha}$ with the Killing spinors, this time on $S^{4}$.

This suggest that one should also be able to write a spinorial version of the fuzzy 4-sphere for some bifundamental matrices $\tilde{G}^{\alpha}$, satisfying

$$
J_{A}=\tilde{G}^{\beta}\left(\Gamma_{A}\right)^{\alpha}{ }_{\beta} \tilde{G}_{\alpha}^{\dagger}, \quad \bar{J}_{A}=\tilde{G}_{\alpha}^{\dagger}\left(\Gamma_{A}\right)^{\alpha}{ }_{\beta} \tilde{G}^{\beta},
$$

where $J_{A}, \bar{J}_{A}$ generate an $\mathrm{SO}(5)$ spinor rotation on $\tilde{G}^{\alpha}$ by

$$
J_{A} \tilde{G}^{\alpha}-\tilde{G}^{\alpha} \bar{J}_{A}=\left(\Gamma_{A}\right)_{\beta}^{\alpha} \tilde{G}^{\beta} .
$$

This in turn implies that the fuzzy sphere should be described by the same GRVV algebra as for the $S^{2}$ case

$$
\tilde{G}^{\alpha}=\tilde{G}^{\alpha} \tilde{G}_{\beta}^{\dagger} \tilde{G}^{\beta}-\tilde{G}^{\beta} \tilde{G}_{\beta}^{\dagger} \tilde{G}^{\alpha}
$$

\footnotetext{
${ }^{13}$ These are constructed as: $\sigma_{1}$ and $\sigma_{3}$ where 1 is replaced by $\mathbb{1}_{2 \times 2}$ and $\sigma_{2}$ where $i$ is replaced by $i \sigma_{1}, i \sigma_{2}, i \sigma_{3}$.
} 
but now with $\tilde{G}^{\alpha}$ being 4 complex matrices that describe a fuzzy 4 -sphere, which poses an interesting possibility that we will however not further investigate here.

$S^{8}$

The third Hopf map, $S^{15} \stackrel{\pi}{\rightarrow} S^{8}$, is related to the octonionic algebra. The $S^{15}$ is expressed now by the real objects $g_{\alpha}^{T} g^{\alpha}=1, \alpha=1, \ldots, 16$ that can be split into two groups $(1, \ldots, 8$ and $9, \ldots, 16)$. The Hopf map is expressed by [39] $\left(g_{\alpha}^{T} g_{\alpha}=1 \Rightarrow x_{A} x_{A}=1\right)$

$$
x_{A}=g_{\alpha}^{T}\left(\Gamma_{A}\right)^{\alpha \beta} g_{\beta},
$$

where $\left(\Gamma_{A}\right)^{\alpha \beta}$ are the $\mathrm{SO}(9)$ gamma-matrices ${ }^{14}$. Similarly for the case of the $S^{4}$ above, even though $g^{\alpha}$ 's are initially 16-dimensional variables acted by the spinor representation of $\mathrm{SO}(9)$, one can project down to the base of the Hopf fibration and replace the $g^{\alpha}$ 's with real 8-dimensional objects on the 8-sphere $\tilde{g}^{\alpha}$. Then the $\tilde{g}^{\alpha}$ 's are identified with the Killing spinors of $S^{8}$.

This once again suggests that one should be able to write a spinorial version of the fuzzy 8-sphere for some bifundamental matrices $\tilde{G}^{\alpha}$ satisfying

$$
J_{A}=\tilde{G}_{\alpha}\left(\Gamma_{A}\right)^{\alpha \beta} \tilde{G}_{\beta}^{T}, \quad \bar{J}_{A}=\tilde{G}_{\alpha}^{T}\left(\Gamma_{A}\right)^{\alpha \beta} \tilde{G}_{\beta},
$$

where $J_{A}, \bar{J}_{A}$ generate an $\mathrm{SO}(9)$ spinor rotation on $\tilde{G}^{\alpha}$ by

$$
J_{A} \tilde{G}_{\alpha}-\tilde{G}_{\alpha} \bar{J}_{A}=\left(\Gamma_{A}\right)_{\alpha}{ }^{\beta} \tilde{G}_{\beta}
$$

and implies the same GRVV algebra, but with the $\tilde{G}^{\alpha}$ 's now being 16 dimensional real matrices that describe the fuzzy 8-sphere.

\section{Deconstruction vs. twisted compactification}

We now describe certain changes which occur when 'deconstructing' a supersymmetric field theory on the bifundamental fuzzy $S^{2}$, in contrast to the usual $S^{2}$, and comparing with the compactified higher-dimensional theory.

The term 'deconstruction' was first coined in [40] for a specific four-dimensional model but more generally extends to creating higher dimensional theories through field theories with matrix degrees of freedom of high rank. In our particular case, the fuzzy $S^{2}$ background arises as a solution in a $d$-dimensional field theory and fluctuations around this background 'deconstruct' a $d+2$-dimensional field theory. We will focus on the case where the $d+2$-dimensional field theory compactified on $S^{2}$ is supersymmetric.

\subsection{Adjoint fuzzy $S^{2}$}

This construction is familiar in the context of D-branes, though any field theory with a fuzzy $S^{2}$ background will also do. For instance, the example we will follow is [41], where an $\mathcal{N}=1$ supersymmetric massive $\mathrm{SU}(N)$ gauge theory around a fuzzy $S^{2}$ background solution, coming

\footnotetext{
${ }^{14}$ The gamma-matrices are constructed similarly to the $S^{4}$ case as follows: $\Gamma_{i}=\left(\begin{array}{cc}0 & \lambda_{i} \\ -\lambda_{i} & 0\end{array}\right), \quad \begin{gathered}\Gamma_{8} \\ =\end{gathered}$ $\left(\begin{array}{cc}0 & \mathbb{1}_{8 \times 8} \\ \mathbb{1}_{8 \times 8} & 0\end{array}\right), \Gamma_{9}=\left(\begin{array}{cc}\mathbb{1}_{8 \times 8} & 0 \\ 0 & -\mathbb{1}_{8 \times 8}\end{array}\right)$, i.e. from $\sigma_{2}$ with $\lambda_{i}$ replacing $i$, and from $\sigma_{1}$ and $\sigma_{3}$ with 1 replaced by $\mathbb{1}_{8 \times 8}$. The $\lambda_{i}$ satisfy $\left\{\lambda_{i}, \lambda_{i}\right\}=-2 \delta_{i j}$ (similarly to the $i \sigma_{i}$ in the case of $S^{4}$ ) and are constructed from the structure constants of the algebra of the octonions [39]. An explicit inversion of the Hopf map is given by $g_{\alpha}=\left[\left(1+x_{9}\right) / 2\right]^{1 / 2} u_{\alpha}$ for $\alpha=1, \ldots, 8$ and $g_{\alpha}=\left[2\left(1+x_{9}\right)\right]^{-1 / 2}\left(x_{8}-x_{i} \lambda_{i}\right) u_{\alpha-8}$ for $\alpha=9, \ldots, 16$, with $u_{\alpha}$ a real 8-component $\mathrm{SO}(8)$ spinor satisfying $u^{\alpha} u_{\alpha}=1$ thus parametrising the $S^{7}$ fibre.
} 
from the low energy theory on a stack of D3-branes in some nontrivial background, was identified with the Maldacena-Núñez theory of IIB 5-branes with twisted compactification on $S^{2}$ [42]. This construction was known to give an $\mathcal{N}=1$ massive theory after dimensional reduction that can be identified with the starting point, thus the D3-brane theory around the fuzzy sphere deconstructs the 5-brane theory.

The twisting of the 5-brane fields can be understood both in the compactification as well as the deconstruction pictures. In compactification, and for the [41] model, it is known from [43] that in order to preserve supersymmetry on D-branes with curved worldvolumes one needs to twist the various D-brane fields. Specifically, that means embedding the $S^{2}$ spin connection, taking values in $\mathrm{SO}(2) \simeq \mathrm{U}(1)$, into the R-symmetry. As a result, the maximal supersymmetry one can obtain after compactification is $\mathcal{N}=1$ (corresponding to $\left.\mathrm{U}(1)_{R}\right)$. On the other hand, in deconstruction, the need for twisting will instead appear by analysing the kinetic operators of the deconstructed fields.

The brane intuition, though useful, is not necessary, and in the following we will understand the twisting as arising generally from requiring supersymmetry of the dimensionally reduced compactified theory. This will be matched by looking at the kinetic term diagonalisation of the deconstructed theory.

\section{Compactification}

On a 2-sphere, scalar fields are decomposed in the usual spherical harmonics $Y_{l m}\left(x_{i}\right)=Y_{l m}(\theta, \phi)$ and can thus give massless fields after compactification (specifically, the $l=0$ modes). However, that is no longer true for spinors and gauge fields. In that case, the harmonic decomposition in terms of $Y_{l m}\left(x_{i}\right)$ must be redefined in order to make explicit the Lorentz properties of spinors and vectors on the 2 -sphere, i.e. to make them eigenvectors of their corresponding operators.

Spinors on the sphere are eigenvectors of the total angular momentum $J_{i}^{2}$. These are of two types: Eigenvectors $\Omega$ of the orbital angular momentum $L_{i}^{2}$ (Cartesian spherical spinors) and eigenvectors $\Upsilon$ of the Dirac operator on the sphere $-i \hat{\nabla}_{S^{2}}=-i \hat{h}^{a b} e_{a}^{m} \sigma_{m} \nabla_{b}$ (spherical basis spinors), whose square is $R^{2}\left(-i \hat{\nabla}_{S^{2}}\right)^{2}=J_{i}^{2}+\frac{1}{4}$. The two are related by a transformation with a sphere-dependent matrix $S$, already described in Section 4.2. The former are decomposed in the spinorial spherical harmonics

$$
\Omega_{j l m}^{\hat{\alpha}}=\sum_{\mu= \pm \frac{1}{2}} C\left(l, \frac{1}{2}, j ; m-\mu, \mu, m\right) Y_{l, m-\mu}(\theta, \phi) \chi_{\mu}^{\hat{\alpha}},
$$

where $j=q_{ \pm}=l \pm \frac{1}{2}$ and $\hat{\alpha}=1,2$, as

$$
\psi^{\hat{\alpha}}=\sum_{l m} \psi_{l m}^{(+)} \Omega_{l+\frac{1}{2}, l m}^{\hat{\alpha}}+\psi_{l m}^{(-)} \Omega_{l-\frac{1}{2}, l m}^{\hat{\alpha}} .
$$

Both have a minimum mass of $\frac{1}{2 R}$, since the Dirac operator squares to $J_{i}^{2}+\frac{1}{4}=j(j+1)+\frac{1}{4}$. Similarly, the vector fields do not simply decompose in $Y_{l m}$ 's, but rather in the vector spherical harmonics

$$
\begin{aligned}
\frac{1}{R} \mathbf{T}_{j m} & =\frac{1}{\sqrt{j(j+1)}}\left[\sin \theta \partial_{\theta} Y_{j m} \hat{\phi}-\csc \theta \partial_{\phi} Y_{j m} \hat{\theta}\right], \\
\frac{1}{R} \mathbf{S}_{j m} & =\frac{1}{\sqrt{j(j+1)}}\left[\partial_{\theta} Y_{j m} \hat{\theta}+\partial_{\phi} Y_{j m} \hat{\phi}\right],
\end{aligned}
$$

with $j \geq 1$. It is more enlightening to show the decomposition of the field strength on the 2-sphere

$$
\frac{1}{R} \csc \theta F_{\theta \phi}=R^{2} \sum_{l m} F_{l m} \frac{1}{\sqrt{l(l+1)}} \Delta_{S^{2}} Y_{l m},
$$


with $l=1,2, \ldots$ Thus again only massive and no massless modes are obtained after dimensional reduction [41]. Note that as we can see, the expansion in spinorial or vector spherical harmonics corresponds to redefining the expansion in terms of $Y_{l m}$ (rearranging its coefficients).

Therefore in the absence of twisting supersymmetry will be lost after dimensional reduction, since all $S^{2}$-fermions will be massive but some massless $S^{2}$-scalars will still remain. Twisting, however, allows for the presence of fermionic twisted-scalars (T-scalars), i.e. fermions that are scalars of the twisted $\mathrm{SO}(2)_{T}$ Lorentz invariance group (with charge $T$ ), which will stay massless. In this way the number of supersymmetries in the dimensionally reduced theory equals the number of fermionic T-scalars.

One chooses the twisted Lorentz invariance of the sphere as $Q_{T}=Q_{x y}+Q_{A}$, where $Q_{x y}$ is the charge under the original Lorentz invariance of the sphere $\mathrm{SO}(2)_{x y}$, and $Q_{A}$ is the charge under the $\mathrm{U}(1)$ subgroup of R-symmetry. This is necessary because one needs to identify the $\mathrm{U}(1)$ spin connection ('gauge field of Lorentz invariance') with a corresponding connection in the R-symmetry subgroup, i.e. a gauge field from the transverse manifold.

An example of an action for twisted fields is provided by the result of [43], for a bosonic T-spinor $\Xi$, fermionic T-scalars $\Lambda$ and T-vectors $g_{a}$

$$
\int d^{d} x d^{2} \sigma \sqrt{h}\left[-\frac{i}{2} \mu \bar{\Lambda} \gamma^{\mu} \partial_{\mu} \Lambda-\frac{i}{2} \mu \bar{g}_{a} \gamma^{\mu} \partial_{\mu} g^{a}+\mu \omega^{a b} \bar{G}_{a b} \Lambda-2 \partial_{\mu} \Xi^{\dagger} \partial^{\mu} \Xi-8 \Xi^{\dagger}\left(-i \hat{\nabla}_{S^{2}}\right)^{2} \Xi\right],
$$

where $\mu$ is the mass parameter, $G_{a b}=\partial_{a} g_{b}-\partial_{b} g_{a}$ is the field strength of the fermionic T-vector, and as usual $\omega^{a b}=\frac{1}{\sqrt{g}} \epsilon^{a b}$ is the symplectic form on the sphere. We note that the kinetic terms in the flat directions $(\mu, \nu)$ are given by their bosonic or fermionic nature, while the type of kinetic terms in the sphere directions $(a, b)$ are dictated by their T-spin and the number of derivatives on it are again dictated by their statistics (bosons have two derivatives, fermions only one).

These fields are decomposed in spherical harmonics corresponding to their T-charge. Then e.g. the fermionic T-scalar can have a massless $(l=0)$ mode, which after dimensional reduction will still be a fermion and give $\mathcal{N}=1$ supersymmetry.

\section{Deconstruction}

To have a fuzzy sphere background of the usual type, we need in the worldvolume theory at least 3 scalar modes $\phi_{i}$ to satisfy $\left[\phi_{i}, \phi_{j}\right]=2 i \epsilon_{i j k} \phi_{k}$, but usually there are more. Then the need for e.g. bosonic T-spinors is uncovered by diagonalising the kinetic term for all the scalar fluctuations around the fuzzy sphere background. For instance in [41], there are 6 scalar modes forming 3 complex scalars $\Phi_{i}$, with fluctuations $\delta \Phi_{i}=a_{i}+i b_{i}$ and kinetic term

$$
\int d^{d} x d^{2} \sigma \sqrt{h} \delta \Phi_{i}^{\dagger}\left[\left(1+J^{2}\right) \delta_{i j}-i \epsilon_{i j k} J_{k}\right] \delta \Phi_{j}
$$

The (complete set of) eigenvectors of this kinetic operator are given by the vector spherical harmonics $J_{i} Y_{l m}$ and the spinorial spherical harmonics $\Omega_{j l m}^{\hat{\alpha}}$. This kinetic operator is then diagonalised by defining T-vectors $n_{a}$ coming from the vector spherical harmonics and T-spinors $\xi^{\hat{\alpha}}$ coming from the spinor spherical harmonics. When completing this program, the deconstructed action is the same as the compactified one, e.g. for [41] one again obtains the twisted action (5.1).

At finite $N$, the matrices are expanded in the fuzzy spherical harmonics $Y_{l m}\left(J_{i}\right)$, becoming the $Y_{l m}\left(x_{i}\right)$ of classical $S^{2}$, but the above diagonalisation corresponds in the classical limit to reorganising the expansion (this includes a nontrivial action on the coefficients of the expansion) to form the spinorial, vector, etc. spherical harmonics.

Thus for the adjoint construction all the fields on the classical $S^{2}$ appear as limits of functions expanded in the scalar fuzzy spherical harmonics, $Y_{l m}\left(J_{i}\right)$, and the various tensor structures of $S^{2}$ fields were made manifest by diagonalising the various kinetic operators. 


\subsection{Bifundamental fuzzy $S^{2}$}

The case of the bifundamental fuzzy $S^{2}$ is richer. One wants to once again compare with the same compactification picture. However, the particulars of the deconstruction will be different.

\section{Deconstruction}

Here we need a fuzzy sphere background of GRVV type, hence at least 2 complex scalar modes $R^{\alpha}$ in the worldvolume theory giving the fuzzy sphere background in terms of $R^{\alpha}=f G^{\alpha}$, with $G^{\alpha}$ satisfying (1.2). The fluctuation of this field will be called $r^{\alpha}$.

Performing the deconstruction follows a set of steps similar to the adjoint fuzzy $S^{2}$, namely one wants to expand in the fuzzy spherical harmonics and in the classical limit reorganise the expansion (acting nontrivially on the coefficients of the expansion) to construct the spinor, vector, etc. spherical harmonics. However now there are some subtle points that one needs to take into account. We have two kinds of fuzzy spherical harmonics, $Y_{l m}\left(J_{i}\right)$ and $Y_{l m}\left(\bar{J}_{i}\right)$, both giving the same $Y_{l m}\left(x_{i}\right)$ in the classical limit. Adjoint fields, e.g. the gauge fields, will be decomposed in terms of one or the other according to their respective gauge groups. On the other hand for bifundamental fields one must first 'extract' a bifundamental GRVV matrix, $\tilde{G}^{\alpha}$ or $\tilde{G}_{a}^{\dagger}$, before one is left with adjoints that can be decomposed in the same way. We detailed this procedure for $r^{\alpha}$ in Section 2.2.3. The expansion in $Y_{l m}\left(x_{i}\right)$ must be then reorganised as in the usual fuzzy $S^{2}$ in order to diagonalise the kinetic operator, thus producing the spinor, vector, etc. spherical harmonics.

The most important difference is that $\tilde{G}^{\alpha}$ has a spinor index on $S^{2}$; in particular we saw in Section 4.2 that in the classical limit $\tilde{g}^{\alpha}$ is identified with a Killing spinor. That means that the operation of 'extracting' $\tilde{G}^{a}$ corresponds to automatically twisting the fields! Let us make this concrete by considering a specific example.

In the mass-deformed ABJM theory, one has besides the $R^{\alpha}$ field a doublet of scalar fields $Q^{\dot{\alpha}}$ with fluctuation $q^{\dot{\alpha}}$, where $\dot{\alpha}$ is an SU(2) index transverse to the sphere. Thus the $q^{\dot{\alpha}}$ start off life as scalars. However, due to their bifundamental nature, one must first 'extract' $\tilde{G}^{\alpha} \rightarrow \sqrt{N} \tilde{g}^{\alpha}$, by writing $q^{\dot{\alpha}}=Q_{\alpha}^{\dot{\alpha}} \tilde{G}^{\alpha}$. In order to diagonalise the kinetic operator, we perform an S-transformation and construct

$$
\Xi_{\dot{\alpha}}^{\alpha}=i\left(P_{+} S^{-1} Q_{\dot{\alpha}}\right)^{\alpha}+\left(P_{-} S^{-1} Q_{\dot{\alpha}}\right)^{\alpha},
$$

after which the kinetic term becomes the twisted action

$$
N^{2} \int d^{3} x d^{2} \sigma \sqrt{\hat{h}}\left[\frac{1}{2} \bar{\Xi}^{\dot{\alpha}}\left(-i 2 \mu \hat{\nabla}_{S^{2}}\right)^{2} \Xi_{\dot{\alpha}}-\frac{1}{2} \partial_{\mu} \bar{\Xi}^{\dot{\alpha}} \partial^{\mu} \Xi_{\dot{\alpha}}-3 \mu^{2} \bar{\Xi}^{\dot{\alpha}} \Xi_{\dot{\alpha}}\right] \text {. }
$$

More generally, the functions on the sphere are actually sections of the appropriate bundle: Either ordinary functions, sections of the spinor or the line bundle. Specifically, anything without an $\alpha$ index is a T-scalar, one $\alpha$ index implies a T-spinor and two $\alpha$ indices a T-scalar plus a T-vector in a $(\mathbf{1} \oplus \mathbf{3})$ decomposition. That is, the $\mathrm{U}(1)_{T}$ invariance is identified with the $\mathrm{SO}(2)_{L} \simeq \mathrm{U}(1)_{L}$ Lorentz invariance of the sphere, described by the index $\alpha$.

In addition to this, an interesting new alternative to the above construction also arises. We can choose to keep $\tilde{G}^{\alpha}$ in the spherical harmonic expansion (by considering it as part of the spherical harmonic in the classical limit). The derivative of the spherical harmonic expansion then includes the derivative of $\tilde{g}^{\alpha}$ given in (4.12) and one obtains a fuzzy version of the classical derivative operator

$$
q_{\dot{\beta}}^{\dagger} J_{i}-\bar{J}_{i} q_{\dot{\beta}}^{\dagger} \rightarrow 2 i K_{i}^{a} \partial_{a} q_{\dot{\beta}}^{\dagger}+q_{\dot{\beta}}^{\dagger} x_{i}
$$


This operator acts on all bifundamental fields, including the ABJM fermions $\psi^{\dagger \alpha}$. In this new kind of expansion, we recover the usual Lorentz covariant kinetic term. For instance for the scalar fields $q^{\dot{\alpha}}$ of ABJM we obtain (after a rescaling of the fields)

$$
\frac{1}{g_{Y M}^{2}} \int d^{3} x d^{2} \sigma \sqrt{h}\left[-\partial^{A} q_{\dot{\alpha}}^{\dagger} \partial_{A} q^{\dot{\alpha}}\right]
$$

where $A=\mu, a$ is a total (worldvolume + fuzzy sphere) index. The price one pays for this simplicity (compared to (5.3)) is however that the classical $N \rightarrow \infty$ limit of the supersymmetry transformation is very subtle, since a naive application will relate fields with different finite $N$ gauge structures (bifundamentals with adjoints), naively implying a gauge-dependent supersymmetry parameter.

But at least formally, by keeping $\tilde{G}^{\alpha}$ inside the spherical harmonic expansion, we obtain an un-twisted, fully supersymmetric version of the action on the whole worldvolume plus the fuzzy sphere.

\section{Supersymmetric D4-brane action on fuzzy $S^{2}$ from ABJM}

As a concrete application of the whole discussion thus far, we present the final results for the Lagrangian obtained by studying fluctuations around the fuzzy $S^{2}$ ground-state of the massdeformed ABJM model.

The fluctuating fields are the $r^{\alpha}$ scalars forming the fuzzy sphere background, transverse scalars $q^{\dot{\alpha}}$, gauge fields $A_{\mu}$ and $\hat{A}_{\mu}$, fermions $\psi_{\alpha}$ and $\chi_{\dot{\alpha}}$. The spherical harmonic expansion on the fuzzy sphere is for each of the above

$$
\begin{aligned}
& r^{\alpha}=r \tilde{G}^{\alpha}+s^{\alpha}{ }_{\beta} \tilde{G}^{\beta}=\left[(r)_{l m} \delta_{\beta}^{\alpha}+\left(s_{\beta}^{\alpha}\right)_{l m}\right] Y_{l m}\left(J_{i}\right) \tilde{G}^{\beta}, \\
& q^{\dot{\alpha}}=Q_{\alpha}^{\dot{\alpha}} \tilde{G}^{\alpha}=\left(Q_{\alpha}^{\dot{\alpha}}\right)_{l m} Y_{l m}\left(J_{i}\right) \tilde{g}^{\alpha}, \\
& \psi_{\alpha}=\tilde{\psi} \tilde{G}_{\alpha}+U_{\alpha}{ }^{\beta} \tilde{G}_{\beta}=\left[(\tilde{\psi})_{l m} \delta_{\alpha}^{\beta}+\left(U_{\alpha}{ }^{\beta}\right)_{l m}\right] Y_{l m}\left(J_{i}\right) \tilde{G}_{\beta}, \\
& \chi_{\dot{\alpha}}=\chi_{\dot{\alpha} \alpha} \tilde{G}^{\alpha}=\left(\chi_{\dot{\alpha} \alpha}\right)_{l m} Y_{l m}\left(J_{i}\right) \tilde{G}^{\alpha}, \\
& A_{\mu}=A_{\mu}^{l m} Y_{l m}\left(J_{i}\right), \quad \hat{A}_{\mu}=\hat{A}_{\mu}^{l m} Y_{l m}\left(\bar{J}_{i}\right),
\end{aligned}
$$

becoming in the classical limit

$$
\begin{aligned}
& r^{\alpha}=r \tilde{g}^{\alpha}+s^{\alpha}{ }_{\beta} \tilde{g}^{\beta}=\left[(r)_{l m} \delta_{\beta}^{\alpha}+\left(s^{\alpha}{ }\right)_{l m}\right] Y_{l m}\left(x_{i}\right) \tilde{g}^{\beta}, \\
& q^{\dot{\alpha}}=Q_{\alpha}^{\dot{\alpha}} \tilde{g}^{\alpha}=\left(Q_{\alpha}^{\dot{\alpha}}\right)_{l m} Y_{l m}\left(x_{i}\right) \tilde{g}^{\alpha}, \\
& \psi_{\alpha}=\tilde{\psi} \tilde{g}_{\alpha}+U_{\alpha}{ }^{\beta} \tilde{g}_{\beta}=\left[(\tilde{\psi})_{l m} \delta_{\alpha}^{\beta}+\left(U_{\alpha}{ }^{\beta}\right)_{l m}\right] Y_{l m}\left(x_{i}\right) \tilde{g}_{\beta}, \\
& \chi_{\dot{\alpha}}=\chi_{\dot{\alpha} \alpha} \tilde{g}^{\alpha}=\left(\chi_{\dot{\alpha} \alpha}\right)_{l m} Y_{l m}\left(x_{i}\right) \tilde{g}^{\alpha}, \\
& A_{\mu}=A_{\mu}^{l m} Y_{l m}\left(x_{i}\right), \quad \hat{A}_{\mu}=\hat{A}_{\mu}^{l m} Y_{l m}\left(x_{i}\right) .
\end{aligned}
$$

These can be further redefined as

$$
s_{\beta}^{\alpha}\left(\tilde{\sigma}_{i}\right)_{\alpha}^{\beta}=K_{i}^{a} A_{a}+x_{i} \phi, \quad \Upsilon_{\dot{\alpha}}^{\alpha}=\left(P_{-} S^{-1} \chi_{\dot{\alpha}}\right)^{\alpha},
$$

with $A_{a}$ becoming the sphere component of the gauge field and $\Phi=2 r+\phi$ becoming a scalar, while $2 r-\phi$ is 'eaten' by the gauge field in a Higgs mechanism that takes us from nonpropagating CS gauge field to propagating YM field in $3 \mathrm{~d}$ [44]. The final supersymmetric version of the action is then

$$
S_{\text {phys }}=\frac{1}{g_{\mathrm{YM}}^{2}} \int d^{3} x d^{2} \sigma \sqrt{h}\left[-\frac{1}{4} F_{A B} F^{A B}-\frac{1}{2} \partial_{A} \Phi \partial^{A} \Phi-\frac{\mu^{2}}{2} \Phi^{2}-\partial^{A} q_{\dot{\alpha}}^{\dagger} \partial_{A} q^{\dot{\alpha}}+\frac{\mu}{2} \omega^{a b} F_{a b} \Phi\right.
$$




$$
\left.+\left(\frac{1}{2} \bar{\Upsilon}^{\dot{\alpha}} \tilde{D}_{5} \Upsilon_{\dot{\alpha}}+\frac{i}{2} \mu \bar{\Upsilon}^{\dot{\alpha}} \Upsilon_{\dot{\alpha}}+\text { h.c. }\right)-(\psi S) \tilde{D}_{5}\left(S^{-1} \psi^{\dagger}\right)+\frac{i}{2} \mu(\psi S)\left(S^{-1} \psi^{\dagger}\right)\right]
$$

The twisting of the fields that have a $\tilde{G}^{\alpha}$ in their spherical harmonic expansion is done as follows: First, we twist by expressing $q^{\dot{\alpha}}$ as $Q_{\alpha}^{\dot{\alpha}}$ and $\psi_{\alpha}$ as $\tilde{\psi}, U_{\alpha}{ }^{\beta}$. We then redefine the twisted fields in order to diagonalise their kinetic operator by further writing $Q_{\alpha}^{\dot{\alpha}}$ according to (5.2) and

$$
\begin{array}{ll}
U_{\alpha}{ }^{\beta}=\frac{1}{2} U_{i}\left(\tilde{\sigma}_{i}\right)_{\alpha}{ }^{\beta}, & \bar{U}_{\alpha}{ }^{\beta}=\frac{1}{2} U_{i}\left(\tilde{\sigma}_{i}\right)_{\alpha}{ }^{\beta}, \\
U_{i}=K_{i}^{a} g_{a}+\hat{\psi} x_{i}, & \bar{U}_{i}=K_{i}^{a} \bar{g}_{a}+\overline{\hat{\psi}} x_{i} .
\end{array}
$$

The final twisted action is

$$
\begin{aligned}
S_{\text {phys }}= & \frac{1}{g_{\mathrm{YM}}^{2}} \int d^{3} x d^{2} \sigma \sqrt{h}\left[-\frac{1}{4} F_{A B} F^{A B}-\frac{1}{2} \partial_{A} \Phi \partial^{A} \Phi-\frac{\mu^{2}}{2} \Phi^{2}+\frac{\mu}{2} \omega^{a b} F_{a b} \Phi\right. \\
& +\left(\frac{1}{2} \bar{\Upsilon}^{\dot{\alpha}} D_{5} \Upsilon_{\dot{\alpha}}+\frac{i}{2} \mu \bar{\Upsilon}^{\dot{\alpha}} \Upsilon_{\dot{\alpha}}+\text { h.c. }\right)+\frac{1}{4} \bar{\Xi}^{\dot{\alpha}}\left(-\frac{2 i}{\mu} \nabla_{S^{2}}\right)^{2} \Xi_{\dot{\alpha}}-\partial_{\mu} \bar{\Xi}^{\dot{\alpha}} \partial^{\mu} \Xi_{\dot{\alpha}} \\
& \left.-\frac{3}{2} \mu^{2} \bar{\Xi}^{\dot{\alpha}} \Xi_{\dot{\alpha}}+\frac{1}{4} \bar{\Lambda} \not \partial \Lambda+\frac{1}{4} \bar{g}_{a} \not \partial g^{a}+\frac{i}{4} \omega^{a b} \bar{G}_{a b} \Lambda+\frac{i}{2} \mu \bar{\Lambda} \Lambda\right] .
\end{aligned}
$$

\section{Conclusions}

In this paper we reviewed our fuzzy $S^{2}$ construction in terms of bifundamental matrices, originally obtained in the context of the ABJM model in [9, 10], focusing on its model-independent mathematical aspects. We found that this is completely equivalent to the usual adjoint $\mathrm{SU}(2)$ construction, but that it involves fuzzy versions of Killing spinors on the 2 -sphere, which we defined. We described the qualitative differences that appear when using the bifundamental $S^{2}$ to 'deconstruct' higher dimensional field theories. The expansion of the fields involving fuzzy Killing spinors result in an automatic twisting of the former on the sphere. Alternatively, including the Killing spinors in the fuzzy spherical harmonic expansion provides a new approach to the construction of fields on $S^{2}$.

We expect that the generality of the construction will lead to it finding a place in numerous applications both in the context of physical systems involving bifundamental matter, e.g. quiver gauge theories as in [45], as well as noncommutative geometry. We hope to further report on both of these aspects in the future.

\section{Acknowledgements}

It is a pleasure to thank Sanjaye Ramgoolam for many comments, discussions and collaboration in [9]. CP is supported by the STFC grant ST/G000395/1.

\section{References}

[1] Myers R.C., Dielectric-branes, J. High Energy Phys. 1999 (1999), no. 12, 022, 41 pages, hep-th/9910053.

[2] Hoppe J., Quantum theory of a massless relativistic surface and a two-dimensional bound state problem, PhD Thesis, Massachusetts Institute of Technology, 1982, available at http://hdl.handle.net/1721.1/15717.

[3] Hoppe J., Diffeomorphism groups, quantization and SU( $\infty)$, Internat. J. Modern Phys. A 4 (1989), 52355248.

[4] Madore J., The fuzzy sphere, Classial Quantum Gravity 9 (1992), 69-88. 
[5] Iso S., Kimura Y., Tanaka K., Wakatsuki K., Noncommutative gauge theory on fuzzy sphere from matrix model, Nuclear Phys. B 604 (2001), 121-147, hep-th/0101102.

[6] Dasgupta K., Sheikh-Jabbari M.M., Van Raamsdonk M., Matrix perturbation theory for M-theory on a PPwave, J. High Energy Phys. 2002 (2002), no. 5, 056, 52 pages, hep-th/0205185.

[7] Dasgupta K., Sheikh-Jabbari M.M., Van Raamsdonk M., Protected multiplets of M-theory on a plane wave, J. High Energy Phys. 2002 (2002), no. 9, 021, 41 pages, hep-th/0207050.

[8] Papageorgakis C., Ramgoolam S., Toumbas N., Noncommutative geometry, quantum effects and DBIscaling in the collapse of D0-D2 bound states, J. High Energy Phys. 2006 (2006), no. 1, 030, 31 pages, hep-th/0510144.

[9] Nastase H., Papageorgakis C., Ramgoolam S., The fuzzy $S^{2}$ structure of M2-M5 systems in ABJM membrane theories, J. High Energy Phys. 2009 (2009), no. 5, 123, 61 pages, arXiv:0903.3966.

[10] Nastase H., Papageorgakis C., Fuzzy Killing spinors and supersymmetric D4 action on the fuzzy 2-sphere from the ABJM model, J. High Energy Phys. 2009 (2009), no. 12, 049, 52 pages, arXiv:0908.3263.

[11] Aharony O., Bergman O., Jafferis D.L., Maldacena J., $N=6$ superconformal Chern-Simons-matter theories, M2-branes and their gravity duals, J. High Energy Phys. 2008 (2008), no. 10, 091, 38 pages, arXiv:0806.1218.

[12] Bagger J., Lambert N., Modeling multiple M2-branes, Phys. Rev. D 75 (2007), 045020, 7 pages, hep-th/0611108.

[13] Bagger J., Lambert N., Gauge symmetry and supersymmetry of multiple M2-branes, Phys. Rev. D 77 (2008), 065008, 6 pages, arXiv:0711.0955.

[14] Bagger J., Lambert N., Comments on multiple M2-branes, J. High Energy Phys. 2008 (2008), no. 2, 105, 15 pages, arXiv:0712.3738.

[15] Gustavsson A., Algebraic structures on parallel M2-branes, Nuclear Phys. B 811 (2009), 66-76, arXiv:0709.1260.

[16] Gustavsson A., Rey S.-J., Enhanced $N=8$ supersymmetry of ABJM theory on $\mathrm{R}(8)$ and $\mathrm{R}(8) / \mathrm{Z}(2)$, arXiv:0906.3568

[17] Kwon O.-K., Oh P., Sohn J., Notes on Supersymmetry Enhancement of ABJM theory, J. High Energy Phys. 2009 (2009), no. 8, 093, 22 pages, arXiv:0906.4333.

[18] Gomis J., Rodríguez-Gómez D., Van Raamsdonk M., Verlinde H., A massive study of M2-brane proposals, J. High Energy Phys. 2008 (2008), no. 9, 113, 29 pages, arXiv:0807.1074.

[19] Hosomichi K., Lee K.-M., Lee S., Lee S., Park J., $N=5,6$ superconformal Chern-Simons theories and M2-branes on orbifolds, J. High Energy Phys. 2008 (2008), no. 9, 002, 24 pages, arXiv:0806.4977.

[20] Bena I., Warner N.P., A harmonic family of dielectric flow solutions with maximal supersymmetry, J. High Energy Phys. 2004 (2004), no. 12, 021, 22 pages, hep-th/0406145.

[21] Lin H., Lunin O., Maldacena J.M., Bubbling AdS space and 1/2 BPS geometries, J. High Energy Phys. 2004 (2004), no. 10, 025, 68 pages, hep-th/0409174.

[22] Terashima S., On M5-branes in $N=6$ membrane action, J. High Energy Phys. 2008 (2008), no. 8, 080, 11 pages, arXiv:0807.0197.

[23] Hanaki K., Lin H., M2-M5 systems in $N=6$ Chern-Simons theory, J. High Energy Phys. 2008 (2008), no. 9, 067, 14 pages, arXiv:0807.2074.

[24] Guralnik Z., Ramgoolam S., On the polarization of unstable D0-branes into non-commutative odd spheres, J. High Energy Phys. 2001 (2001), no. 2, 032, 17 pages, hep-th/0101001.

[25] Ramgoolam S., On spherical harmonics for fuzzy spheres in diverse dimensions, Nuclear Phys. B 610 (2001), 461-488, hep-th/0105006.

[26] Ramgoolam S., Higher dimensional geometries related to fuzzy odd-dimensional spheres, J. High Energy Phys. 2002 (2002), no. 10, 064, 29 pages, hep-th/0207111.

[27] Van Raamsdonk M., Comments on the Bagger-Lambert theory and multiple M2-branes, J. High Energy Phys. 2008 (2008), no. 5, 105, 9 pages, arXiv:0803.3803.

[28] Hasebe K., Kimura Y., Fuzzy supersphere and supermonopole, Nuclear Phys. B 709 (2005), 94-114, hep-th/0409230.

[29] Grosse H., Reiter G., The fuzzy supersphere, J. Geom. Phys. 28 (1998), 349-383, math-ph/9804013.

[30] Balachandran A.P., Kurkcuoglu S., Vaidya S., Lectures on fuzzy and fuzzy SUSY physics, hep-th/0511114. 
[31] van Nieuwenhuizen P., An introduction to simple supergravity and the Kaluza-Klein program, in Relativity, Groups and Topology, II (Les Houches, 1983), North-Holland, Amsterdam, 1984, 823-932.

[32] Eastaugh A., van Nieuwenhuizen P., Harmonics and spectra on general coset manifolds, Kyoto Summer Institute 1985:0001, Preprint ITP-SB-85-43.

[33] van Nieuwenhuizen P., The complete mass spectrum of $d=11$ supergravity compactified on $S_{4}$ and a general mass formula for arbitrary cosets $M_{4}$, Classial Quantum Gravity 2 (1985), 1-20.

[34] Gunaydin M., van Nieuwenhuizen P., Warner N.P., General construction of the unitary representations of anti-de Sitter superalgebras and the spectrum of the $S^{4}$ compactification of 11-dimensional supergravity, Nuclear Phys. B 255 (1985), 63-92.

[35] Nastase H., Vaman D., van Nieuwenhuizen P., Consistency of the $\operatorname{AdS}_{7} \times S_{4}$ reduction and the origin of self-duality in odd dimensions, Nuclear Phys. B 581 (2000), 179-239, hep-th/9911238.

[36] Van Nieuwenhuizen P., Supergravity, Phys. Rep. 68 (1981), 189-398.

[37] Kim H.J., Romans L.J. , van Nieuwenhuizen P., Mass spectrum of chiral ten-dimensional $N=2$ supergravity on $S^{5}$, Phys. Rev. D 32 (1985), 389-399.

[38] Wu Y.S., Zee A., Membranes, higher Hopf maps, and phase interactions, Phys. Lett. B 207 (1988), 39-43.

[39] Bernevig B.A., Hu J.-P., Toumbas N., Zhang S.-C., Eight-dimensional quantum hall effect and "octonions", Phys. Rev. Lett. 91 (2003), 236803, 4 pages, cond-mat/0306045.

[40] Arkani-Hamed N., Cohen A.G., Georgi H., (De)constructing dimensions, Phys. Rev. Lett. 86 (2001), 47574761, hep-th/0104005.

[41] Andrews R.P., Dorey N., Deconstruction of the Maldacena-Núñez compactification, Nuclear Phys. B 751 (2006), 304-341, hep-th/0601098.

[42] Maldacena J.M., Nuñez C., Supergravity description of field theories on curved manifolds and a no go theorem, Internat. J. Modern Phys. A 16 (2001), 822-855, hep-th/0007018.

[43] Bershadsky M., Vafa C., Sadov V., D-branes and topological field theories, Nuclear Phys. B 463 (1996), 420-434, hep-th/9511222.

[44] Mukhi S., Papageorgakis C., M2 to D2, J. High Energy Phys. 2008 (2008), no. 5, 085,15 pages, arXiv:0803.3218.

[45] Maldacena J., Martelli D., The unwarped, resolved, deformed conifold: fivebranes and the baryonic branch of the Klebanov-Strassler theory, arXiv:0906.0591. 